Bull. Soc. math. France

129 (4), 2001, p. 547-591

\title{
ON THE PARTIAL ALGEBRAICITY \\ OF HOLOMORPHIC MAPPINGS BETWEEN TWO REAL ALGEBRAIC SETS
}

\author{
BY JoËL MERKER
}

\begin{abstract}
The rigidity properties of the local invariants of real algebraic CauchyRiemann structures imposes upon holomorphic mappings some global rational properties (Poincaré 1907) or more generally algebraic ones (Webster 1977). Our principal goal will be to unify the classical or recent results in the subject, building on a study of the transcendence degree, to discuss also the usual assumption of minimality in the sense of Tumanov, in arbitrary dimension, without rank assumption and for holomorphic mappings between two arbitrary real algebraic sets.

RÉSUMÉ (Algébricité partielle des applications holomorphes entre deux ensembles algébriques réels)

La rigidité des invariants locaux des structures de Cauchy-Riemann réelles algébriques impose aux applications holomorphes des propriétés globales de rationalité (Poincaré 1907), ou plus généralement d'algébricité (Webster 1977). Notre objectif principal sera d'unifier les résultats classiques ou récents, grâce à une étude du degré de transcendance, de discuter aussi l'hypothèse habituelle de minimalité au sens de Tumanov, et ce en dimension quelconque, sans hypothèse de rang et pour des applications holomorphes quelconques entre deux ensembles algébriques réels arbitraires.
\end{abstract}

Texte reçu le 20 décembre 1999, révisé le 4 septembre 2000 et le 30 janvier 2001, accepté le 7 mars 2001

JoËL Merker, Laboratoire d'Analyse, Topologie et Probabilités, Centre de Mathématiques et d'Informatique, UMR 6632, 39 rue Joliot Curie, 13453 Marseille Cedex 13 (France)

E-mail : merkercmi.univ-mrs.fr

2000 Mathematics Subject Classification. - 32V25, 32V40, 32V15, 32V10.

Key words and phrases. - Local holomorphic mappings, Real algebraic sets, Transcendence degree, Local algebraic foliations, Minimality in the sense of Tumanov, Segre chains. 


\section{Introduction}

The algebraicity or the rationality of local holomorphic mappings between real algebraic CR manifolds can be considered to be one of the most remarkable phenomena in CR geometry. Introducing the consideration of Segre varieties in the historical article [17], Webster generalized the classical rationality properties of self-mappings between three-dimensional spheres discovered by Poincaré and later extended by Tanaka to arbitrary dimension. Webster's theorem states that biholomorphisms between Levi non-degenerate real algebraic hypersurfaces in $\mathbb{C}^{n}$ are algebraic. Around the eighties, some authors studied proper holomorphic mappings between spheres of different dimensions or between pieces of strongly pseudoconvex real algebraic hypersurfaces, notably Pelles, Alexander, Fefferman, Pinchuk, Chern-Moser, Diederich-Fornaess, Faran, Cima-Suffridge, Forstnerič, Sukhov, and others (complete references are provided in [2], [5], [8], [13], [15], [17], [18]). In the past decade, removing the equidimensionality condition in the classical theorem of Webster, Sukhov for mappings between Levi non-degenerate quadrics [15], Huang [8] for mappings between strongly pseudoconvex hypersurfaces, and Sharipov-Sukhov [13] for mappings between general Levi non-degenerate real algebraic CR manifolds have exhibited various sufficient conditions for the algebraicity of a general local holomorphic map $f: M \rightarrow M^{\prime}$ between two real algebraic CR manifolds $M \subset \mathbb{C}^{n}$ and $M^{\prime} \subset \mathbb{C}^{n^{\prime}}$. A necessary and sufficient condition, but with a rank condition on $f$ is provided in [2]. Recently, using purely algebraic methods, Coupet-Meylan-Sukhov ( $c f$. [5]; see also [6]) have estimated the transcendence degree of $f$ directly. Building on their work, we aim essentially to study the algebraicity question in full generality ( $c f$. Problem 2.4) and to unify the various approaches of [2], [5], [8], [12], [13], [17], [18]. Notably, we shall state necessary and sufficient conditions for the algebraicity of $f$ without rank condition and we shall study the geometry of the minimality assumption thoroughly.

\section{Presentation of the main result}

2.1. Algebraicity of holomorphic mappings and their transcendence degree. - Let $U \subset \mathbb{C}^{n}$ be a small nonempty open polydisc. A holomorphic mapping $f: U \rightarrow \mathbb{C}^{n^{\prime}}, f \in \mathcal{H}\left(U, \mathbb{C}^{n^{\prime}}\right)$, is called algebraic if its graph is contained in an irreducible $n$-dimensional complex algebraic subset of $\mathbb{C}^{n} \times \mathbb{C}^{n^{\prime}}$. Using classical elimination theory, one can show that, equivalently, each of its components $g:=f_{1}, \ldots, f_{n^{\prime}}$ satisfies a nontrivial polynomial equation $g^{r} a_{r}+\cdots+a_{0}=0$, the $a_{j} \in \mathbb{C}[z]$ being polynomials. We recall that a set $\Sigma \subset U$ is called real algebraic if it is given as the zero set in $U$ of a finite number of real algebraic polynomials in $\left(z_{1}, \ldots, z_{n}, \bar{z}_{1}, \ldots, \bar{z}_{n}\right)$.

Let us denote by $\mathcal{A}=\mathbb{C}[z]$ the ring of complex polynomial functions over $\mathbb{C}^{n}$ and by $\mathcal{R}=\mathbb{C}(z)$ its quotient field $\operatorname{Fr}(\mathcal{A})$. By $\mathcal{R}\left(f_{1}, \ldots, f_{n^{\prime}}\right)$ we understand the 
field generated by $f_{1}, \ldots, f_{n^{\prime}}$ over $\mathcal{R}$, which is a subfield of the field of meromorphic functions over $U$ and which identifies with the collection of rational functions

$$
R\left(f_{1}, \ldots, f_{n^{\prime}}\right)=P\left(f_{1}, \ldots, f_{n^{\prime}}\right) / Q\left(f_{1}, \ldots, f_{n^{\prime}}\right),
$$

$P, Q \in \mathcal{R}\left[x_{1}, \ldots, x_{n^{\prime}}\right]$ and $Q \neq 0$.

Following [5], the transcendence degree $\nabla^{\operatorname{tr}}(f)$ of the field $\mathcal{R}\left(f_{1}, \ldots, f_{n^{\prime}}\right)$ with respect to the field $\mathcal{R}$ provides an integer-valued invariant measuring the lack of algebraicity of $f$. In particular, $\nabla^{\operatorname{tr}}(f)$ is zero if and only if $f$ is algebraic.

Indeed, by definition $\nabla^{\operatorname{tr}}(f)$ coincides with the maximal cardinal number $\kappa^{\prime}$ of a subset

$$
\left\{f_{j_{1}}, \ldots, f_{j_{\kappa^{\prime}}}\right\} \subset\left\{f_{1}, \ldots, f_{n^{\prime}}\right\}, \quad 1 \leq j_{1}<\cdots<j_{\kappa^{\prime}} \leq n^{\prime}
$$

which is algebraically independent over $\mathcal{R}$. In other words, $\nabla^{\operatorname{tr}}(f)=\kappa^{\prime}$ means that there exists a subset $\left\{f_{j_{1}}, \ldots, f_{j_{\kappa^{\prime}}}\right\} \subset\left\{f_{1}, \ldots, f_{n^{\prime}}\right\}$ such that there does not exist a nontrivial relation

$$
P\left(f_{j_{1}}, \ldots, f_{j_{\kappa^{\prime}}}\right) \equiv 0 \text { in } \mathcal{H}(U), \quad P \in \mathcal{R}\left[x_{1}, \ldots, x_{\kappa^{\prime}}\right] \backslash\{0\},
$$

but that for every $\lambda, \kappa^{\prime}+1 \leq \lambda \leq n^{\prime}$, every $1 \leq j_{1}<\cdots<j_{\lambda} \leq n^{\prime}$, there exists an algebraic relation $Q\left(f_{j_{1}}, \ldots, f_{j_{\lambda}}\right) \equiv 0, Q \in \mathcal{R}\left[x_{1}, \ldots, x_{\lambda}\right] \backslash\{0\}$. Of course,

$$
\nabla^{\operatorname{tr}}\left(f_{1}, \ldots, f_{n^{\prime}}\right) \leq n^{\prime}
$$

An equivalent geometric characterization of $\nabla^{\operatorname{tr}}(f)$ states that $\nabla^{\operatorname{tr}}(f)=\kappa^{\prime}$ if and only if the dimension of the minimal for inclusion complex algebraic set $\Lambda_{f} \subset U \times \mathbb{C}^{n^{\prime}}$ containing the graph

$$
\Gamma_{f}=\left\{(z, f(z)) \in U \times \mathbb{C}^{n^{\prime}}: z \in U\right\}
$$

of $f$ is equal to $n+\kappa^{\prime}$ (this complex algebraic set $\Lambda_{f}$ is of course necessarily irreducible). In other words, $\nabla^{\operatorname{tr}}(f)$ is an invariant intrinsically attached to $f$ which is given with $f$ and which possesses an algebraic and a geometric signification. In a metaphoric sense, we can think that $\nabla^{\operatorname{tr}}(f)$ measures the lack of algebraicity of $f$, or conversely, that it provides an estimation of the maximal partial algebraicity properties of $f$.

2.3. Presentation of the main result. - Then, because the transcendence degree is an appropriate invariant, more general than the dichotomy between algebraic and non-algebraic objects, we shall as in [5] study directly the transcendence degree of holomorphic mappings between two real algebraic sets. Our main goal is to provide a synthesis of the results in [2], [5], [8], [13], [17], [18]. Thus, quite generally, let $f: U \rightarrow \mathbb{C}^{n^{\prime}}$ be a local holomorphic mapping sending an arbitrary irreducible real algebraic set $\Sigma \cap U$ into another real algebraic set $\Sigma^{\prime} \subset \mathbb{C}^{n^{\prime}}$. As the algebraicity of $f$ is a non-local property, we shall assume in the sequel that $\Sigma \cap U$ is a smooth closed CR-submanifold of $U$ and 
that there exists a second polydisc $U^{\prime} \subset \mathbb{C}^{n^{\prime}}$ with $f(U) \subset U^{\prime}$ such that $\Sigma^{\prime} \cap U^{\prime}$ is also a smoooth closed CR-submanifold of $U^{\prime}$. We shall denote by $M$ and $M^{\prime}$ these connected local CR pieces of $\Sigma$ in $U$ and of $\Sigma^{\prime}$ in $U^{\prime}$. Of course, after shrinking again $U$ and $U^{\prime}$, we can suppose that $f$ is of constant rank over $U$. The topic of this article is to study in full generality the following problem by seeking an optimal bound:

Problem 2.4. - Estimate $\nabla^{\operatorname{tr}}(f)$ in terms of geometric invariants of $f, \Sigma, \Sigma^{\prime}$.

To begin with, we shall first assume that $M$ is somewhere minimal in the sense of Tumanov, as in [2], [5], [8], [13], [17], [18]. In the sequel, we shall say that a property $\mathcal{P}$ holds at a Zariski-generic point $p \in \Sigma$ if there exist a proper real algebraic subset $E$ of $\Sigma$, depending on $\mathcal{P}$, such that the property $\mathcal{P}$ holds at each point of $\Sigma \backslash E$. Let $\Delta$ be the unit disc in $\mathbb{C}$. Our main result lies in the following statement from which we shall recover every algebraicity result of the cited literature.

THEOREM 2.5. - Assume that $M$ is CR-generic, connected and minimal in the sense of Tumanov at a Zariski-generic point, and let $\Sigma^{\prime \prime}$ be the minimal (for inclusion, hence irreducible) real algebraic set satisfying $f(M) \subset \Sigma^{\prime \prime} \subset \Sigma^{\prime}$. Let $\kappa^{\prime}$ denote the transcendence degree $\nabla^{\operatorname{tr}}(f)$ of $f$. Then near a Zariski-generic point $p^{\prime \prime} \in \Sigma^{\prime \prime}$, there exists a local algebraic coordinate system in which $\Sigma^{\prime \prime}$ is of the form $\Delta^{\kappa^{\prime}} \times \underline{\Sigma}^{\prime \prime}$ for some real algebraic variety $\Sigma^{\prime \prime} \subset \mathbb{C}^{n^{\prime}-\kappa^{\prime}}$.

This theorem says that the degree of nonalgebraicity of $f$ imposes some degeneracy condition on $\Sigma^{\prime}$, namely to contain a smaller real algebraic set $\Sigma^{\prime \prime}$ which is 'degenerate' in the sense that it can be locally straightened to be a product by a complex $\nabla^{\operatorname{tr}}(f)$-dimensional polydisc at almost every point. The main interest of Theorem 2.5 lies in fact in its various reciprocal forms which are listed in $\S 3$ below. Of course, the assumption that $\nabla^{\operatorname{tr}}(f)$ equals an integer $\kappa^{\prime}$ is no assumption at all, since $\nabla^{\operatorname{tr}}(f)$ is automatically given with $f$, but in truth abstractly, namely in a non-constructive way, as is $\Sigma^{\prime \prime}$. The only unjustified assumption with respect to Problem 2.4 is the minimality of $M$ in the sense of Tumanov and it remains also to explain why the estimate given by Theorem 2.5 is sharp and satisfactory.

Thus, let us firstly consider the sharpness. If $\Sigma^{\prime \prime}$ is an irreducible real algebraic set as above, it can be shown that there exists the largest integer $\kappa_{\Sigma^{\prime \prime}}$ such that $\Sigma^{\prime \prime}$ is a product of the form $\Delta^{\kappa_{\Sigma^{\prime \prime}}} \times \Sigma^{\prime \prime}$ near a Zariski-generic point in suitable local algebraic coordinates (see Theorem 9.10). This integer will also be abbreviated by $\kappa_{\Sigma^{\prime \prime}}$ and we shall say that $\Sigma^{\prime \prime}$ is $\kappa_{\Sigma^{\prime \prime}}$-algebraically degenerate. We shall also write

$$
\Sigma^{\prime \prime} \cap V^{\prime \prime} \cong \Delta_{\mathcal{A}} \Delta^{\kappa^{\prime \prime}} \times \underline{\Sigma}^{\prime \prime}
$$

to mean that $\Sigma^{\prime \prime}$ intersected with the small open set $V^{\prime \prime}$ is equivalent to a product in complex algebraic (abbreviation: $\mathcal{A}$ ) coordinates. Then Theorem 2.5

TOME $129-2001-\mathrm{N}^{\mathrm{O}} 4$ 
states in summary that $\kappa_{\Sigma^{\prime \prime}} \geq \nabla^{\operatorname{tr}}(f)$. With this notion defined and this rephrasing of Theorem 2.5 at hand, we now notice that it can of course well happen that $\kappa_{\Sigma^{\prime \prime}}>\nabla^{\operatorname{tr}}(f)$. For instance, this happens when $n=n^{\prime}$, when $f$ is an algebraic biholomorphic map, so $\nabla^{\operatorname{tr}}(f)=0$, and when $\Sigma=\Sigma^{\prime}=\Sigma^{\prime \prime}=\mathbb{C}^{n}$ simply. So what? In case where $\kappa_{\Sigma^{\prime \prime}}>\nabla^{\operatorname{tr}}(f)$, by an elementary observation we shall show that a suitable perturbation of $f$ can raise and maximize its possible transcendence degree. The precise statement, which establishes the desired sharpness, is as follows.

TheOrem 2.6. - Assume that $f$ is nonconstant and that $\Sigma^{\prime \prime}$ is the minimal for inclusion real algebraic set satisfying $f(M) \subset \Sigma^{\prime \prime} \subset \Sigma^{\prime}$. Remember that $\Sigma^{\prime \prime}$ is locally equivalent to $\Delta^{\kappa} \Sigma^{\prime \prime} \times \Sigma^{\prime \prime}$ at a Zariski-generic point. Then there exist a point $p \in M$ such that $f(p)=: p^{\prime \prime} \in \Sigma^{\prime \prime}$ is a Zariski-generic point of $\Sigma^{\prime \prime}$ and a local holomorphic self-map $\phi$ of $\Sigma^{\prime \prime}$ fixing $p^{\prime \prime}$ which is arbitrarily close to the identity map, such that $\nabla^{\operatorname{tr}}(\phi \circ f)=\kappa_{\Sigma^{\prime \prime}}$ (of course, because of Theorem 2.5, it is impossible to produce $\left.\nabla^{\operatorname{tr}}(\phi \circ f)>\kappa_{\Sigma^{\prime \prime}}\right)$.

Secondly, let us discuss the (until now still unjustified) minimality in the sense of Tumanov assumption. Remember that CR manifold without infinitesimal CR automorphisms are quite poor, since they carry few self-maps. In $\S 13.2$ below, we shall observe the following.

THEOREM 2.7. - Let $M$ be a nowhere minimal real algebraic CR-generic manifold and assume that the space of infinitesimal CR-automorphisms of $M$ is nonzero. Then $M$ admits a local one-parameter family of nonalgebraic biholomorphic self-maps.

\section{Five corollaries}

The direct converse of the main Theorem 2.5 bounds $\nabla^{\operatorname{tr}}(f)$ as follows and gives an optimal sufficient condition for $f$ to be algebraic.

Theorem 3.1. - Let $f \in \mathcal{H}\left(U, \mathbb{C}^{n^{\prime}}\right)$ with $f(M) \subset \Sigma^{\prime}$ and assume $M$ is $C R$ generic and minimal at a Zariski-generic point. Then $\nabla^{\operatorname{tr}}(f) \leq \kappa_{\Sigma^{\prime \prime}}=$ algebraic degeneracy degree of the minimal for inclusion real algebraic set $\Sigma^{\prime \prime}$ such that $f(M) \subset \Sigma^{\prime \prime} \subset \Sigma^{\prime}$. In particular, $f$ is necessarily algebraic if there does not exist an 1-algebraically degenerate real algebraic set $\Sigma^{\prime \prime \prime}$ with $f(M) \subset \Sigma^{\prime \prime \prime} \subset \Sigma^{\prime}$.

As Theorem 2.6 shows that, otherwise, $f$ can be slightly perturbed to be nonalgebraic, this theorem provides a necessary and sufficient condition for $f$ to be algebraic. Thanks to this synthetic general converse, we will recover results of the cited litterature as corollaries. We also obtain as a corollary the celebrated algebraicity result in [17] from the following statement.

BULletin DE LA SOCIÉtÉ MATHÉMATIQUE DE FRANCE 
Corollary 3.2 (see [2]). — Any biholomorphism between pieces of smooth real algebraic CR-generic holomorphically nondegenerate manifolds in $\mathbb{C}^{n}$ which are minimal at a Zariski-generic point must be algebraic.

Proof. - Let $f: M \rightarrow M^{\prime}$ biholomorphic. Suppose by contradiction that $\nabla^{\operatorname{tr}}(f)=\kappa^{\prime} \geq 1$. We prove in this paper (Corollary 9.14) that $M^{\prime}$ is holomorphically degenerate if and only if $M^{\prime}$ is algebraically degenerate. Since $f(M) \equiv M^{\prime}$ is locally onto, necessarily $M^{\prime \prime}=M^{\prime}$ is $\kappa^{\prime}$-holomorphically degenerate, by Theorem 2.5, a contradiction.

For instance, here is another more general consequence of Theorem 3.1. By $\mathcal{V}_{\mathbb{C}^{n}}(p)$, we denote a small open polydisc centered at $p$ whose size may shrink. Let $\Sigma_{\text {reg }}^{\prime}$ denote the regular part of $\Sigma^{\prime}$ in the sense of real algebraic geometry.

Corollary 3.3. - Let $f \in \mathcal{H}\left(U, \mathbb{C}^{n^{\prime}}\right)$ with $f(M) \subset \Sigma^{\prime}$ and assume $M$ is CR-generic and minimal at a Zariski-generic point. If

$$
f\left(M \cap \mathcal{V}_{\mathbb{C}^{n}}(p)\right) \supset \mathcal{V}_{\mathbb{C}^{n^{\prime}}}\left(f(p) \cap \Sigma_{\mathrm{reg}}^{\prime}\right)
$$

for some point $p \in M$, then $\Sigma^{\prime \prime}=\Sigma^{\prime}$ and $\nabla^{\operatorname{tr}}(f) \leq \kappa_{\Sigma^{\prime}}$.

Recall now that a CR-generic manifold $M$ is called Segre-transversal at $p \in M$ if for all $V=\mathcal{V}_{\mathbb{C}^{n}}(p)$, there exists $k \in \mathbb{N}_{*}$ and $q_{1}, \ldots, q_{k} \in V \cap S_{\bar{p}}$ such that

$$
T_{p} S_{\bar{q}_{1}}+\cdots+T_{p} S_{\bar{q}_{k}}=T_{p} \mathbb{C}^{n}
$$

the $S_{\bar{r}}$ denoting Segre varieties, hence $p \in S_{\bar{q}_{1}}, \ldots, p \in S_{\bar{q}_{k}}$. Segre-transversal $M$ 's are always minimal. In fact, Segre-transversality at $p \in M$ appears to be equivalent to minimality at $p$ (see [5]) in codimension 1 , when $M$ is a hypersurface, but in codimension $\geq 3$ there exist already some minimal and not Segre-transversal $(M, p)$ 's. Since the question whether minimal CR-generic manifolds $(M, p)$ of codimension 2 are Segre transversal was left open in [5], we devote $\S 10$ to answer it affirmatively. Now, another important and direct consequence of our main Theorem 2.5 is the following statement, implying in particular [8].

Corollary 3.4 (see [5]). - Let $f \in \mathcal{H}\left(U, \mathbb{C}^{n^{\prime}}\right)$ with $f(M) \subset \Sigma^{\prime}$ and assume that $M$ is Segre-transversal at $p$. Then

$$
\begin{aligned}
\nabla^{\operatorname{tr}}(f) \leq m^{\prime}:= & \text { the maximal dimension of a complex algebraic variety } A_{f(q)}^{\prime} \\
& \text { with } f(q) \in A_{f(q)}^{\prime} \subset \Sigma^{\prime}, \text { where } q \text { runs in } M .
\end{aligned}
$$

Proof. - First, $M$ is minimal at $p$ (see [5]; see also $\S 14$ here). Clearly, if $\kappa^{\prime}=$ $\nabla^{\operatorname{tr}}(f)$, then $\Sigma^{\prime}$ contains a piece of algebraic set $A^{\prime} \cong_{\mathcal{A}} \Delta^{\kappa^{\prime}}$ by Theorem 2.5, so $\kappa^{\prime} \leq m^{\prime}$.

A fourth consequence of Theorem 3.1 is:

TOME $129-2001-\mathrm{N}^{\mathrm{O}} 4$ 
Corollary 3.5 (see [18]). - Let $f \in \mathcal{H}\left(U, \mathbb{C}^{n^{\prime}}\right)$ with $f(M \cap U) \subset \Sigma^{\prime}$ and assume that $M$ is minimal at a Zariski-generic point. If $\Sigma^{\prime}$ does not any contain complex algebraic varieties, then $\nabla^{\operatorname{tr}}(f)=0$, i.e. $f$ is algebraic.

Proof. - As above, if $\kappa^{\prime}=\nabla^{\operatorname{tr}}(f) \geq 1$, then $M^{\prime} \supset A^{\prime} \cong_{\mathcal{A}} \Delta^{\kappa^{\prime}}$.

In conclusion, we therefore unify the results in the papers [2], [5], [8], [13], [17], [18].

3.6. Second reflection. - Let $f: M \rightarrow M^{\prime}$,

$$
M: \rho(z, \bar{z})=0, \quad 0 \in M, \quad M^{\prime}: \rho^{\prime}\left(z^{\prime}, \bar{z}^{\prime}\right)=0, \quad 0 \in M^{\prime},
$$

$\rho, \rho^{\prime}$ real algebraic polynomials: $\rho \in \mathbb{C}[z, \bar{z}]^{d}, \rho^{\prime} \in \mathbb{C}\left[z^{\prime}, \bar{z}^{\prime}\right]^{d^{\prime}}$,

$$
d=\operatorname{codim}_{\mathbb{R}} M, \quad d^{\prime}=\operatorname{codim}_{\mathbb{R}} M^{\prime},
$$

and let $S_{\bar{w}}, S_{\bar{w}^{\prime}}^{\prime}$ denote Segre varieties,

$$
\begin{gathered}
w \in \mathcal{V}_{\mathbb{C}^{n}}(0):=U, \quad w^{\prime} \in \mathcal{V}_{\mathbb{C}^{n^{\prime}}}(0):=U^{\prime} \\
S_{\bar{w}}:=\{z \in U: \rho(z, \bar{w})=0\}, \quad S_{\bar{w}^{\prime}}^{\prime}:=\left\{z^{\prime} \in U^{\prime}: \rho^{\prime}\left(z^{\prime}, \bar{w}^{\prime}\right)=0\right\} .
\end{gathered}
$$

Following [7], [10], [18], for every subset $E^{\prime} \subset U^{\prime}$, we define the first and the second reflection of $E^{\prime}$ (which appears in an article of Diederich-Fornaess, Ann. Math. 107 (1978), pp. 371-384) by

$$
r_{M^{\prime}}\left(E^{\prime}\right):=\left\{w^{\prime} \in U^{\prime}: S_{\bar{w}^{\prime}}^{\prime} \supset E^{\prime}\right\}, \quad r_{M^{\prime}}^{2}\left(E^{\prime}\right):=r_{M^{\prime}}\left(r_{M^{\prime}}\left(E^{\prime}\right)\right) .
$$

One establishes easily that given $f: M \rightarrow M^{\prime}$, then (see [10]):

$$
f(z) \in \mathbb{X}_{z, \bar{w}}^{\prime}:=r_{M^{\prime}}\left(f\left(S_{\bar{z}}\right)\right) \cap r_{M^{\prime}}^{2}\left(f\left(S_{\bar{w}}\right)\right) .
$$

Although the paper [18] contains a slightly different statement using the double reflection determination (3.8), we shall summarize its main theorem by the following statement:

$$
\left\langle\operatorname{dim}_{\mathbb{C}} \mathbb{X}_{z, \bar{w}}^{\prime}=0, \forall z, w \in \mathcal{V}_{\mathbb{C}^{n}}(0), \text { with } z \in S_{\bar{w}}\right\rangle \Longrightarrow \nabla^{\operatorname{tr}}(f)=0 .
$$

In fact, to review the version [10], the condition $\operatorname{dim}_{\mathbb{C}} \mathbb{X}_{z, \bar{w}}^{\prime}=0$ for all $z, w \in$ $\mathcal{V}_{\mathbb{C}^{n}}(0), z \in S_{\bar{w}}$, yields easily that $f$ is algebraic on every Segre variety $S_{\bar{w}}$ and it suffices afterwards to apply Theorem 5.1 below to get $\nabla^{\operatorname{tr}}(f)=0$. But the main point here is again that, contrary to the one in Theorem 2.5, this condition (3.9) is only sufficient. Indeed, it appears that there is no natural reason why there should exist a nonalgebraic perturbation $\phi \circ f$ as in Theorem 2.6 in case where $\operatorname{dim}_{\mathbb{C}} \mathbb{X}_{z, \bar{w}}^{\prime} \geq 1$ for all $z, w, z \in S_{\bar{w}}$. For instance, there are cases where $\operatorname{dim}_{\mathbb{C}} \mathbb{X}_{z, \bar{w}}^{\prime} \geq 1$ for all $z, w \in \mathcal{V}_{\mathbb{C}^{n}}(0), z \in S_{\bar{w}}$, but a suitable modification of the method in [7], [10], [18] yields another determinacy set $\mathbb{M}_{z, \bar{w}}^{\prime}$ such that $f(z) \in \mathbb{M}_{z, \bar{w}}^{\prime}$ again, such that $\operatorname{dim}_{\mathbb{C}} \mathbb{M}_{z, \bar{w}}^{\prime}=0$ for all $z, w \in \mathcal{V}_{\mathbb{C}^{n}}(0), z \in S_{\bar{w}}$ and such that, moreover, $\mathbb{M}_{z, \bar{w}}^{\prime}$ is also appropriate for showing that $f$ is algebraic as in (3.9) above. By examples similar to the ones in [10], it can be shown that neither the condition $\operatorname{dim}_{\mathbb{C}} \mathbb{X}_{z, \bar{w}}^{\prime} \geq 1$ for all $z, w \in \mathcal{V}_{\mathbb{C}^{n}}(0), z \in S_{\bar{w}}$, 
nor $\operatorname{dim}_{\mathbb{C}} \mathbb{M}_{z, \bar{w}}^{\prime} \geq 1$ for all $z, w \in \mathcal{V}_{\mathbb{C}^{n}}(0), z \in S_{\bar{w}}$ mean that we can find a nonalgebraic perturbation $\phi \circ f$ : both conditions are only sufficient. Further, the preprints [7], [10] explains the unexpected phenomena which are caused by the action of second reflection $r_{M^{\prime}}^{2}$. By an examination of the examples in [10], the reader can realize that the trouble in them comes from the fact that $M^{\prime}$ is not a priori a piece of the minimal for inclusion real algebraic set $\Sigma^{\prime \prime}$ containing $f(M)$. And we can add that these puzzling phenomena are essentially due to the fact that $r_{M^{\prime}}$ reverses inclusion of sets: $E^{\prime} \subset F^{\prime}$ implies $r_{M^{\prime}}\left(E^{\prime}\right) \supset r_{M^{\prime}}\left(F^{\prime}\right)$ and for this reason, $E^{\prime} \cap r_{M^{\prime}}\left(E^{\prime}\right)$ and $F^{\prime} \cap r_{M^{\prime}}\left(F^{\prime}\right)$ cannot be comparable a priori. For instance, if $f(M) \subset M^{\prime \prime} \subset M^{\prime}$ are as above, one has $r_{M^{\prime}}\left(f\left(S_{\bar{z}}\right)\right) \subset r_{M^{\prime \prime}}\left(f\left(S_{\bar{z}}\right)\right)$ but the two double reflection sets

$$
\begin{aligned}
& \mathbb{X}_{z, \bar{w}}^{\prime}:=r_{M^{\prime}}\left(f\left(S_{\bar{z}}\right)\right) \cap r_{M^{\prime}}^{2}\left(f\left(S_{\bar{w}}\right)\right) \text { and } \\
& \mathbb{X}_{z, \bar{w}}^{\prime \prime}:=r_{M^{\prime \prime}}\left(f\left(S_{\bar{z}}\right)\right) \cap r_{M^{\prime \prime}}^{2}\left(f\left(S_{\bar{w}}\right)\right)
\end{aligned}
$$

are not comparable in general. Explicit examples showing that one can play very freely with these inclusions are given in [7], [10]. This shows that it is more natural to have a condition about $\mathbb{X}_{z, \bar{w}}^{\prime \prime}$ with $M^{\prime \prime}$ being a piece of the minimal for inclusion real algebraic set $\Sigma^{\prime \prime}$ which is smooth. We can of course assume smoothness after shrinking $M$ and $U$ a little bit, since if otherwise $f(M)$ is contained in the (real algebraic) singular part of $\Sigma^{\prime \prime}$ then $\Sigma^{\prime \prime}$ is not minimal for inclusion. Finally, after assuming that $M^{\prime}$ is already minimal for inclusion, we can derive quickly from our Theorem 2.5 the contraposition of Theorem 1.1 in [18].

Corollary 3.11. - Let $f \in \mathcal{H}\left(U, \mathbb{C}^{n^{\prime}}\right), f(M) \subset M^{\prime}$, with $M$ CR-generic and minimal at a Zariski-generic point. Assume that $M^{\prime}$ is minimal for inclusion real algebraic $C R$-generic containing $f(M)$ and smooth. If $\nabla^{\operatorname{tr}}(f) \geq 1$, then there exists $D_{M} \subset M \cap U$ a Zariski-open subset of $M$ such that for all $p \in D_{M}$,

$$
\operatorname{dim}_{\mathbb{C}} \mathbb{X}_{z, \bar{w}}^{\prime} \geq \nabla^{\operatorname{tr}}(f) \geq 1
$$

for all $z, w \in \mathcal{V}_{\mathbb{C}^{n}}(p), z \in S_{\bar{w}}$. Equivalently, if $\operatorname{dim}_{\mathbb{C}} \mathbb{X}_{z, \bar{w}}^{\prime}=0$ on an open set, then $f$ is algebraic. In particular, if $\operatorname{dim}_{\mathbb{C}} r_{M^{\prime}}\left(f\left(S_{\bar{z}}\right)\right)=0$ on a open set, then $f$ is algebraic.

Proof. - Let $\kappa^{\prime}:=\nabla^{\operatorname{tr}}(f)$. By Theorem 2.5, $M^{\prime}$ is at least $\kappa^{\prime}$-algebraically degenerate, i.e. $\kappa_{M^{\prime}} \geq \kappa^{\prime}$. Then

$$
\left(M^{\prime}, p^{\prime}\right) \cong{ }_{\mathcal{A}} \Delta^{\kappa_{M^{\prime}}} \times \underline{M}^{\prime}
$$

locally in a neighborhood of a Zariski-generic point $p^{\prime} \in M^{\prime}$. Let $D_{M}$ be the open set of points $p \in M \cap U$ such that $M^{\prime} \cap \mathcal{V}_{\mathbb{C}^{n^{\prime}}}(f(p)) \cong_{\mathcal{A}} \Delta^{\kappa_{M^{\prime}}} \times \underline{M}^{\prime}$. Since $M^{\prime}$ is minimal for inclusion, we claim that $D_{M}$ is Zariski-open in $M \cap U$. Indeed, otherwise, $f(M \cap U)$ would be contained in the set of points $p^{\prime} \in M^{\prime}$ where $M^{\prime} \cap \mathcal{V}_{\mathbb{C}^{n^{\prime}}}\left(p^{\prime}\right) \oiint_{\mathcal{A}} \Delta^{\kappa_{M^{\prime}}} \times \underline{M}^{\prime}$, which is a proper real algebraic subvariety of $M^{\prime}$

TOME $129-2001-\mathrm{N}^{\mathrm{O}} 4$ 
see Theorems 9.10 and 9.16 below, contradicting the choice of $M^{\prime}$. Assume therefore that $M^{\prime}=\Delta^{\kappa_{M^{\prime}}} \times \underline{M}^{\prime}$ in $U^{\prime}=\mathcal{V}_{\mathbb{C}^{n}}(0)$. Let

$$
\pi^{\prime}: \mathbb{C}^{n^{\prime}} \longrightarrow \mathbb{C}^{n^{\prime}-\kappa_{M^{\prime}}} \times 0
$$

be the projection. It is easy to show that for an arbitrary set $E^{\prime} \subset U^{\prime}$, we have

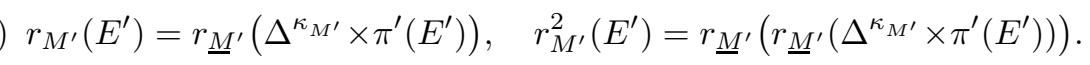

Consequently, $\mathbb{X}_{z, \bar{w}}^{\prime} \supset \Delta^{\kappa_{M^{\prime}}} \times \pi^{\prime}(f(z))$ and

$$
\operatorname{dim}_{\mathbb{C}} \mathbb{X}_{z, \bar{w}}^{\prime} \geq \kappa_{M^{\prime}} \geq \kappa^{\prime}=\nabla^{\operatorname{tr}}(f) \geq 1
$$

3.12. Summary of the proof of Theorem 2.5. - Let $\nabla^{\operatorname{tr}}(f)=: \kappa^{\prime}$. Equivalently, the graph of $f$ is contained in an algebraic $\left(n+\kappa^{\prime}\right)$-dimensional manifold in $\mathbb{C}^{n} \times \mathbb{C}^{n^{\prime}}$ and some $\kappa^{\prime}$ components of $f$, say $\left(f_{1}, \ldots, f_{\kappa^{\prime}}\right):=f_{\left(\kappa^{\prime}\right)}$, make a transcendence basis of the field extension $\mathcal{R} \rightarrow \mathcal{R}\left(f_{1}, \ldots, f_{n^{\prime}}\right)$. By the assumption $f(M) \subset \Sigma^{\prime}$, there exist algebraic relations between $f$ and $\bar{f}$ and then between $f_{\left(\kappa^{\prime}\right)}$ and $\bar{f}_{\left(\kappa^{\prime}\right)}$, after elimination. The main argument in $\S 4$ below shows that an algebraic dependence $R^{\prime}\left(z, \bar{z}, f_{\left(\kappa^{\prime}\right)}(z), \bar{f}_{\left(\kappa^{\prime}\right)}(\bar{z})\right) \equiv 0$, for $z \in M$, can be transformed into an algebraic dependence $S^{\prime}\left(z, f_{\left(\kappa^{\prime}\right)}\right) \equiv 0$ which does not involve the antiholomorphic components in case $(M, p)$ is minimal. This fact strongly relies on the Theorem 8.2 about propagation of partial algebraicity along the Segre surfaces of $(M, p)$. Then $S^{\prime} \equiv 0$, and this will show easily that the set $\Sigma^{\prime \prime}$ is $\kappa^{\prime}$-algebraically degenerate.

3.13. Organization of the article. - Paragraph 4 provides the proof of Theorem 2.5, except the theorem on propagation of algebraicity, to which $\S \S 5-8$ are devoted. Paragraph 7 contains the proof of Theorem 2.7. Paragraph 9 presents the notion of algebraic degeneracy. Paragraph 10 studies the notion of Segre-transversality in the real algebraic context. Finally, Paragraph 11 shows how to produce a statement equivalent to our main Theorem 2.5 using the so-called reflection mapping.

3.14. Acknowledgement. - Since I use transcendence degree in this paper, I would like to thank Bernard Coupet, Francine Meylan and Alexander Sukhov, who kindly provided me with their joint work in February 1998, and I wish to address special thanks to Alexander for several interesting discussions. Also, I have benefited of fruitful conversations about the jet method with Bernard Coupet and Sylvain Damour. Finally, I wish to thank the referee for his clever help. 


\section{Estimate of transcendence degree}

\subsection{Necessity in the main theorem}

We proceed here first to the proof of the easy Theorem 2.6. Thanks to the existence of algebraic stratifications and thanks to delocalization (i.e. choice of a smaller $U$ centered at Zariski-generic point), we reduce the problem to a nonconstant holomorphic map $f: M \rightarrow \Delta^{\kappa^{\prime}} \times \underline{\Sigma}^{\prime \prime}, \kappa^{\prime} \geq 1$. To begin with, assume first that $f$ is algebraic, that $p=0$ and $f(p)=0$. We write

$$
f=\left(\underline{f}_{1}, \ldots, \underline{f}_{n^{\prime \prime}}, f_{1}, \ldots, f_{\kappa^{\prime}}\right) .
$$

Obviously, all perturbations $\left(\underline{f}_{1}, \ldots, \underline{f}_{n^{\prime \prime}}, g_{1}, \ldots, g_{\kappa^{\prime}}\right)$ of $f$, where the map

$$
g: \mathcal{V}_{\mathbb{C}^{n}}(0) \longrightarrow \Delta^{\kappa^{\prime}}, \quad g(0)=0
$$

is an arbitrary holomorphic map, still send $M$ into $\Delta^{\kappa^{\prime}} \times \underline{\Sigma}^{\prime \prime}$. We need to find such a perturbation of the form $\phi \circ f$ with transcendence degree $\kappa^{\prime}$.

Because $\Sigma^{\prime \prime}$ is assumed to be minimal for inclusion containing $f(M)$, we have $f_{1} \not \equiv 0$. Next, we choose a transcendent entire holomorphic function with high order of vanishing

$$
\varpi: \Delta \longrightarrow \Delta, \quad \varpi(0)=0,
$$

say for instance $\varpi\left(z_{1}\right)=\left(\sin z_{1}\right)^{a}, a \in \mathbb{N}_{*}, a \gg 1$, and we define the map $\phi: \Delta^{\kappa^{\prime}} \times \underline{\Sigma}^{\prime \prime} \rightarrow \Delta^{\kappa^{\prime}} \times \underline{\Sigma}^{\prime \prime}$ by

$$
\begin{array}{r}
\phi\left(\underline{z}_{1}, \ldots, \underline{z}_{n^{\prime \prime}}, z_{1}, \ldots, z_{\kappa^{\prime}}\right):=\left(\underline{z}_{1}, \ldots, \underline{z}_{n^{\prime \prime}}, z_{1}+\varpi\left(z_{1}\right), z_{2}+\varpi^{\circ 2}\left(z_{1}\right), \ldots,\right. \\
\left.z_{\kappa^{\prime}}+\varpi^{\circ \kappa^{\prime}}\left(z_{1}\right)\right),
\end{array}
$$

where we denote

$$
\varpi^{\circ k}:=\varpi \circ \cdots \circ \varpi \quad(k \text { times })
$$

Then we have

$$
\nabla^{\operatorname{tr}}\left(\varpi, \varpi^{\circ 2}, \ldots, \varpi^{\circ k}\right)=k
$$

for all $k \in \mathbb{N}_{*}$ and

$$
\phi \circ f=\left(\underline{f}_{1}, \ldots, \underline{f}_{n^{\prime \prime}}, f_{1}+\varpi\left(f_{1}\right), \ldots, f_{\kappa^{\prime}}+\varpi^{\circ \kappa^{\prime}}\left(f_{1}\right)\right) .
$$

If $f$ was algebraic, then clearly $\nabla^{\operatorname{tr}}(\phi \circ f) \geq \kappa^{\prime}$ and the rank at $0 \in \mathbb{C}^{n}$ (or the generic rank) of $f$ is preserved after composition by $\phi$, because $\phi$ is arbitrarily close to the identity map in a neighborhood of the origin.

Now, if $f$ was not algebraic, we choose instead functions

$$
\varpi_{1}, \ldots, \varpi_{\kappa^{\prime}}: \Delta \longrightarrow \Delta
$$

with high order of vanishing at 0 such that

$$
\nabla^{\operatorname{tr}}\left(f_{1}+\varpi_{1}\left(f_{1}\right), f_{2}+\varpi_{2}\left(f_{1}\right), \ldots, f_{\kappa^{\prime}}+\varpi_{\kappa^{\prime}}\left(f_{1}\right)\right)=\kappa^{\prime},
$$

which is possible. Then again $\nabla^{\operatorname{tr}}(\phi \circ f) \geq \kappa^{\prime}$.

TOME $129-2001-\mathrm{N}^{\mathrm{O}} 4$ 


\subsection{Sufficiency in the main theorem}

We establish here Theorem 2.5. This paragraph will essentially follow the lines of the main argument given in [5]. Let $\kappa^{\prime}:=\nabla^{\operatorname{tr}}(f)$ and choose a point $p \in M$ at which $M$ is minimal such that $\Sigma^{\prime \prime}$ is smooth (in the sense of real algebraic geometry) and CR at $f(p)$. This choice can be simply achieved by avoiding some two proper real algebraic subvarieties in the source and in the target. Of course, the real algebraic set $\Sigma^{\prime \prime}$ which is minimal for inclusion satisfying $f(M) \subset \Sigma^{\prime \prime} \subset \Sigma^{\prime}$ is irreducible and $f(M)$ is not contained in $\Sigma_{\text {sing. }}^{\prime \prime}$. Suppose we have proved that $\Sigma^{\prime \prime}$ is $\kappa^{\prime}$-algebraically at $f(p)$. Then Theorem 9.10 below yields that $\Sigma_{\mathrm{reg}, \mathrm{CR}}^{\prime \prime}$ is $\kappa^{\prime}$-algebraically degenerate everywhere. We are thus reduced to prove Theorem 2.6 at one point. To summarize, thanks to the above simplifications, it is clear that it suffices now to establish the following local statement.

ThEOREM 4.3. - Assume that $f:(M, p) \rightarrow\left(M^{\prime}, p^{\prime}\right)$ is a holomorphic map of constant rank between two smooth CR-generic small manifold pieces of real algebraic sets and let $\kappa^{\prime}:=\nabla^{\operatorname{tr}}(f)$. If $(M, p)$ is minimal and $\left(M^{\prime}, p^{\prime}\right)$ minimal for inclusion containing $f(M, p)$, then $\left(M^{\prime}, p^{\prime}\right)$ is at least $\kappa^{\prime}$-algebraically degenerate.

Proof. - For basic definitions about algebraic or transcendental extensions that will be needed in this demonstration, we refer the reader to $\S 2$ of [5] and the references therein.

If $k$ is a subfield of a field $E$, then $E$ is said to be an extension field of $k$. We write $k \rightarrow E$. We only recall the following important lemma.

LEMMA 4.4. - Let $E=k\left(\alpha_{1}, \ldots, \alpha_{n}\right)$ be a finite extension of a field $k$, $n \in \mathbb{N}_{*}$, consisting of rational functions of $\left(\alpha_{1}, \ldots, \alpha_{n}\right)$ with coefficients in $k$. Then $\nabla^{\operatorname{tr}}(k \rightarrow E)=\kappa^{\prime} \geq 1$ if and only if, after renumbering:

1) $\alpha_{1}$ is transcendent over $k, \ldots, \alpha_{\kappa^{\prime}}$ is transcendent over $k\left(\alpha_{1}, \ldots, \alpha_{\kappa^{\prime}-1}\right)$;

2) $\alpha_{\kappa^{\prime}+1}, \ldots, \alpha_{n}$ are algebraic over $k\left(\alpha_{1}, \ldots, \alpha_{\kappa^{\prime}}\right)$.

Let $\mathcal{R}:=\operatorname{Fr}(\mathcal{A})=\mathbb{C}(z)$ denote the quotient field of the ring of algebraic functions $\mathcal{A}:=\mathbb{C}[z]$ over $\mathbb{C}^{n}$ and let us consider $\mathcal{R}\left(f_{1}, \ldots, f_{n^{\prime}}\right)$, the field of rational functions generated by the components $f_{1}, \ldots, f_{n^{\prime}}$ of $f$, i.e. by fractions of the form

$$
\frac{P\left(f_{1}, \ldots, f_{n^{\prime}}\right)}{Q\left(f_{1}, \ldots, f_{n^{\prime}}\right)}
$$

with

$$
P\left(f_{1}, \ldots, f_{n^{\prime}}\right)=\sum_{J} a_{J} f^{J}, \quad Q\left(f_{1}, \ldots, f_{n^{\prime}}\right)=\sum_{J} b_{J} f^{J},
$$

$P$ and $Q$ being polynomials with coefficients $a_{J} \in \mathcal{R}, b_{J} \in \mathcal{R}$. If $\kappa^{\prime}$ denotes the transcendence degree of the field extension $\mathcal{R} \rightarrow \mathcal{R}(f)$, one can assume 
(after renumbering) that $f_{1}, \ldots, f_{\kappa^{\prime}}$ is the basis of transcendence, which means (cf. 4.4)

1) $f_{1}$ is transcendent over $\mathcal{R}, \ldots, f_{\kappa^{\prime}}$ is transcendent over $\mathcal{R}\left(f_{1}, \ldots, f_{\kappa^{\prime}-1}\right)$;

2) $f_{\kappa^{\prime}+1}, \ldots, f_{n^{\prime}}$ are algebraic over $\mathcal{R}\left(f_{1}, \ldots, f_{\kappa^{\prime}}\right)$.

In particular 1) implies that there are no algebraic relations between $\left(f_{1}, \ldots, f_{\kappa^{\prime}}\right)$. Let us denote $f_{\left(\kappa^{\prime}\right)}:=\left(f_{1}, \ldots, f_{\kappa^{\prime}}\right)$. Also, 2$)$ means that there are irreducible monic polynomials

$$
S_{\kappa^{\prime}+1}^{\prime}\left(z, f_{\left(\kappa^{\prime}\right)} ; X\right) \in \mathcal{R}\left(f_{\left(\kappa^{\prime}\right)}\right)[X], \ldots, S_{n^{\prime}}^{\prime}\left(z, f_{\left(\kappa^{\prime}\right)} ; X\right) \in \mathcal{R}\left(f_{\left(\kappa^{\prime}\right)}\right)[X]
$$

such that writing

$$
S_{j}^{\prime}=\sum_{0 \leq k \leq m_{j}} S_{j k}^{\prime}\left(z, f_{\left(\kappa^{\prime}\right)}\right) X^{m_{j}-k}, \quad S_{j 0}^{\prime}=1,
$$

one has

$$
S_{j}^{\prime}\left(z, f_{\left(\kappa^{\prime}\right)} ; f_{j}(z)\right) \equiv 0
$$

identically for $z \in U, j=\kappa^{\prime}+1, \ldots, n^{\prime}$ with $S_{j k}^{\prime}\left(z, f_{\left(\kappa^{\prime}\right)}\right) \in \mathcal{R}\left(f_{\left(\kappa^{\prime}\right)}\right)$. In other words, the graph of $f$

$$
\begin{aligned}
\Gamma(f)=\Gamma\left(f_{1}, \ldots, f_{n^{\prime}}\right)=\left\{\left(z, z^{\prime}\right)\right. & \in \mathbb{C}^{n} \times \mathbb{C}^{n^{\prime}} \\
& \left.z_{1}^{\prime}=f_{1}(z), \ldots, z_{n^{\prime}}^{\prime}=f_{n^{\prime}}(z), z \in U\right\}
\end{aligned}
$$

is contained in the complex algebraic set

$$
\begin{aligned}
\Lambda^{\prime}=\left\{\left(z, z^{\prime}\right) \in \mathbb{C}^{n} \times \mathbb{C}^{n^{\prime}} ; S_{j}^{\prime}\left(z, z_{\left(\kappa^{\prime}\right)}^{\prime} ; z_{j}^{\prime}\right)=0,\right. \\
\\
\left.j=\kappa^{\prime}+1, \ldots, n^{\prime}, z \in U, z^{\prime} \in U^{\prime}\right\} \subset \mathbb{C}^{n} \times \mathbb{C}^{n^{\prime}},
\end{aligned}
$$

equipped with a natural projection $\tau^{\prime}: \mathbb{C}^{n} \times \mathbb{C}^{n^{\prime}} \rightarrow \mathbb{C}^{n} \times \mathbb{C}^{\kappa^{\prime}}$, which is a local algebraic biholomorphism

$$
\tau^{\prime}: \Lambda^{\prime} \backslash\left(\tau^{\prime}\right)^{-1}\left(\Upsilon^{\prime}\right) \longrightarrow \mathbb{C}^{n} \times \mathbb{C}^{\kappa^{\prime}}
$$

outside the inverse image $\left(\tau^{\prime}\right)^{-1}\left(\Upsilon^{\prime}\right)$ of the union $\Upsilon^{\prime}$ of the discriminant loci of the irreducible polynomials $S_{j}^{\prime}$ (hence $\Upsilon^{\prime}$ is a complex algebraic subset of $\mathbb{C}^{n} \times \mathbb{C}^{\kappa^{\prime}}$ of dimension $\left.\leq n+\kappa^{\prime}-1\right)$.

Consider now the graph of the transcendent basis

$$
\begin{aligned}
\Gamma\left(f_{1}, \ldots, f_{\kappa^{\prime}}\right)=\left\{\left(z, z_{\left(\kappa^{\prime}\right)}^{\prime}\right)\right. & \in \mathbb{C}^{n} \times \mathbb{C}^{\kappa^{\prime}} ; \\
& \left.z_{1}^{\prime}=f_{1}(z), \ldots, z_{\kappa^{\prime}}^{\prime}=f_{\kappa^{\prime}}(z), z \in U\right\} .
\end{aligned}
$$

LEMMA 4.11. - For any nonempty open set $V_{M} \subset M$, one has

$$
\Gamma\left(f_{1}, \ldots, f_{\kappa^{\prime}}\right)_{\mid V_{M}} \not \subset \Upsilon^{\prime} .
$$

Proof. - Assuming $V_{M}=V \cap M$ with both $V \subset \mathbb{C}^{n}$ and $V_{M} \subset M$ connected, it would imply $\Gamma\left(f_{1}, \ldots, f_{\kappa^{\prime}}\right)_{\mid V} \subset \Upsilon^{\prime}$ (identity principle), so there would be a nontrivial algebraic relation between $f_{1}, \ldots, f_{\kappa^{\prime}}$.

TOME $129-2001-\mathrm{N}^{\mathrm{O}} 4$ 
After delocalization, we then have $\left(p, f_{\left(\kappa^{\prime}\right)}(p)\right) \notin \Upsilon^{\prime}$ for all $p \in U$, so the complex algebraic set $\Lambda^{\prime}$ in $U \times U^{\prime}$ can be locally defined by equations of the form

$$
z_{\kappa^{\prime}+1}^{\prime}=h_{\kappa^{\prime}+1}^{\prime}\left(z, z_{1}^{\prime}, \ldots, z_{\kappa^{\prime}}^{\prime}\right), \ldots, z_{n^{\prime}}^{\prime}=h_{n^{\prime}}^{\prime}\left(z, z_{1}^{\prime}, \ldots, z_{\kappa^{\prime}}^{\prime}\right)
$$

using the algebraic implicit function theorem, where the $h_{j}^{\prime}$ are holomorphic algebraic functions, i.e. holomorphic functions whose graph is contained in a complex algebraic set of dimension $n+\kappa^{\prime}$.

By hypothesis, $M^{\prime}$ is given by real polynomial equations $P_{j}^{\prime}\left(z^{\prime}, \bar{z}^{\prime}\right)=0$, $j=1, \ldots, \sigma^{\prime}$, near $p^{\prime}$ and we have $P_{j}^{\prime}(f(z), \bar{f}(\bar{z})) \equiv 0$ for $z \in M$. As in classical elimination theory, we can replace the variables $z_{\kappa^{\prime}+1}^{\prime}, \bar{z}_{\kappa^{\prime}+1}^{\prime}, \ldots, z_{n^{\prime}}^{\prime}, \bar{z}_{n^{\prime}}^{\prime}$ by the above values (4.13) and their conjugate values (the $h_{j}^{\prime}$ satisfy polynomial equations, so consider the other 'conjugate' roots of these polynomials), take the product of these equations, use Newton's identities and get that $\pi^{\prime}(f(M))=f_{\left(\kappa^{\prime}\right)}(M)$ is contained in a real algebraic set with polynomial equations $R_{j}^{\prime}\left(z, \bar{z}, z_{1}^{\prime}, \ldots, z_{\kappa^{\prime}}^{\prime}, \bar{z}_{1}^{\prime}, \ldots, \bar{z}_{\kappa^{\prime}}^{\prime}\right)=0, j=1, \ldots, \sigma^{\prime}$. We thus have

$$
R_{j}^{\prime}\left(z, \bar{z}, f_{\left(\kappa^{\prime}\right)}(z), \bar{f}_{\left(\kappa^{\prime}\right)}(\bar{z})\right) \equiv 0, \quad j=1, \ldots, \sigma^{\prime}, z \in M .
$$

In summary, insofar we have eliminated the relatively algebraic components $f_{\kappa^{\prime}+1}, \ldots, f_{n^{\prime}}$ and we are now left with some algebraic relations between the transcendence basis and its conjugate, namely (4.14) above. The crucial Proposition 4.16 below shows that all $R_{j}^{\prime} \equiv 0$ necessarily. We then claim that this fact will readily imply that $M^{\prime}$ contains (and is equal, by minimality for inclusion) a $\kappa^{\prime}$-algebraically degenerate set like in Theorems 2.5 and 4.3.

Indeed, as $R_{j}^{\prime} \equiv 0,1 \leq j \leq \sigma^{\prime}$, then for each $z \in M$, the $\kappa^{\prime}$-dimensional algebraic manifold

$$
A_{z}^{\prime}=\left\{z_{j}^{\prime}=h_{j}^{\prime}\left(z, z_{\left(\kappa^{\prime}\right)}\right), j=\kappa^{\prime}+1, \ldots, n^{\prime}\right\}
$$

is contained in $M^{\prime}$. Let $\pi^{\prime}:\left(z, z^{\prime}\right) \mapsto z^{\prime}$. Then the set of complex algebraic $A_{z}^{\prime}$ parameterized by $z \in U$

$$
\mathcal{C}=\left\{\left(z, z_{\left(\kappa^{\prime}\right)}^{\prime}, h_{\left(n^{\prime}-\kappa^{\prime}\right)}^{\prime}\left(z, z_{\left(\kappa^{\prime}\right)}^{\prime}\right)\right)\right\} \subset U \times U^{\prime}
$$

algebraically projects via $\pi^{\prime}$ into $M^{\prime}$, whenever $z \in M$. The fibers of $\pi^{\prime} \mid \mathcal{C}$ only depend on $z$, so there exists an algebraic submanifold $N$ of $M$ where $\pi^{\prime}$ has constant rank and $\pi^{\prime}: \mathcal{C} \cap\left(N \times U^{\prime}\right) \rightarrow M^{\prime}$ is an algebraic real diffeomorphism by minimality of $M^{\prime}$ for inclusion. Let $Q_{j}(z, \bar{z})=0, j=1, \ldots, \mu$ be some local real polynomial equations for $N$. As $\mathcal{C} \cap\left(N \times U^{\prime}\right)$ is given by the equations

$$
Q_{j}(z, \bar{z})=0, \quad z_{\left(n^{\prime}-\kappa^{\prime}\right)}^{\prime}=h_{\left(n^{\prime}-\kappa^{\prime}\right)}^{\prime}\left(z, z_{\left(\kappa^{\prime}\right)}^{\prime}\right)
$$

the local algebraic biholomorphism defined by

$$
\tilde{z}_{\left(n^{\prime}-\kappa^{\prime}\right)}:=z_{\left(n^{\prime}-\kappa^{\prime}\right)}^{\prime}-h_{\left(n^{\prime}-\kappa^{\prime}\right)}^{\prime}\left(z, z_{\left(\kappa^{\prime}\right)}^{\prime}\right), \quad \tilde{z}_{\left(\kappa^{\prime}\right)}^{\prime}:=z_{\left(\kappa^{\prime}\right)}^{\prime}, \quad \tilde{z}:=z
$$

BULLETIN DE LA SOCIÉTÉ MATHÉMATIQUE DE FRANCE 
clearly straightens $\mathcal{C} \cap\left(N \times U^{\prime}\right)$ to be the product of the real algebraic manifold

$$
\left\{\left(\tilde{z}, \tilde{z}_{\left(n^{\prime}-\kappa^{\prime}\right)}^{\prime}\right): Q_{j}(\tilde{z}, \overline{\tilde{z}})=0, j=1, \ldots, \mu, \tilde{z}_{\left(n^{\prime}-\kappa^{\prime}\right)}^{\prime}=0\right\}
$$

by the $\kappa^{\prime}$-dimensional local polydisc $\left\{\left(0, \tilde{z}_{\left(\kappa^{\prime}\right)}^{\prime}, 0\right)\right\}$. As $\pi^{\prime}: \mathcal{C} \cap\left(N \times U^{\prime}\right) \rightarrow M^{\prime}$ is an algebraic CR-diffeomorphism, this shows that $M^{\prime}$ is at least $\kappa^{\prime}$-algebraically degenerate. Granted Proposition 4.16 below, then Theorem 4.3 will be proved.

It remains to show that no algebraic relation can be satisfied by the transcendence basis $f_{\left(\kappa^{\prime}\right)}$ together with its conjugate $\bar{f}_{\left(\kappa^{\prime}\right)}$. This is where the reflection principle and the minimality of $M$ come on scene.

Proposition 4.16. - Let $g:=\left(g_{1}, \ldots, g_{\kappa^{\prime}}\right)$, with $g_{j}(z)$ holomorphic in $U$. Assume that $\nabla^{\operatorname{tr}}(g)=\kappa^{\prime}$. Then any polynomial relation satisfied by $g$ and $\bar{g}$ over the field of rational functions is trivial, namely

$$
R(z, \bar{z}, g(z), \bar{g}(\bar{z})) \equiv 0, z \in M, \quad \text { implies } \quad R \equiv 0 .
$$

Proof. — Let us proceed by contradiction. Then there exists an integer $\mu \in \mathbb{N}_{*}$, such that we can write

$$
R(z, \bar{z}, g, \bar{g})=\sum_{1 \leq i \leq \mu} g^{\alpha_{i}} r_{i}(z, \bar{z}, \bar{g})
$$

where $\alpha_{i} \in \mathbb{N}^{\kappa^{\prime}}$ and where $r_{i} \neq 0$ for $i=1, \ldots, \mu$ and such that

$$
R(z, \bar{z}, g(z), \bar{g}(\bar{z})) \equiv 0, \quad \text { for } z \in M \text {. }
$$

Here, $R$ and the $r_{i}$ 's are polynomial. Of course, we can assume that $\mu$ is the minimal integer for such a property. We shall use the minimality of $\mu$ to derive the contradiction. Here, since the $r_{i}(z, \bar{z}, \bar{g})$ are nonzero polynomials in $(z, \bar{z}, \bar{g})$, then for $z$ running in $U$, the terms $r_{i}(z, \bar{z}, \bar{g}(\bar{z}))$ (pull-back to $M$ ) can be considered, after obvious reordering, as polynomials in $z$ with coefficients being holomorphic functions of $\bar{z}$ (we loose algebraicity in $\bar{z}$, because the terms $\bar{g}(\bar{z}$ ) are only holomorphic). We can thus write them as

$$
r_{i}(\bar{z} ; z):=\sum_{J} a_{i, J}(\bar{z}) z^{J}
$$

where such a sum is understood to be finite. By minimality of $\mu$, necessarily no $r_{i}(\bar{z} ; z)$ vanishes identically on $M$. For $i=2, \ldots, \mu$, we can set

$$
t_{i}(\bar{z} ; z):=r_{i}(\bar{z} ; z) / r_{1}(\bar{z} ; z),
$$

which are terms of the form $\sum_{J} a_{i, J}(\bar{z}) z^{J} /\left(\sum_{J} a_{1, J}(\bar{z}) z^{J}\right)$ and each sum is finite. Then we have the following relation for $z$ running over the Zariski open subset $D_{M}:=\left\{r_{1} \neq 0\right\}$ of $M$ :

$$
g^{\alpha_{1}}(z)+\sum_{2 \leq i \leq \mu} t_{i}(\bar{z} ; z) g^{\alpha_{i}}(z) \equiv 0, \quad z \in D_{M} .
$$

TOME $129-2001-\mathrm{N}^{\mathrm{O}} 4$ 
Now, let $\bar{L}_{1}, \ldots, \bar{L}_{m}$ be a basis of $T^{0,1} M$ with polynomial coefficients, where $m=\operatorname{dim}_{\mathrm{CR}} M$. Of course, since $g(z)$ is holomorphic, $\bar{L}_{k}\left(g_{i}\right) \equiv 0$ on $M$, for all $1 \leq k \leq m$ and $1 \leq i \leq \kappa^{\prime}$. Applying these CR derivations to (4.17), we then see that the term $g^{\alpha_{1}}(z)$ is automatically killed. We therefore come to a dichotomy. Either $\bar{L}_{k}\left(t_{i}(\bar{z} ; z)\right) \equiv 0$, for all $1 \leq k \leq m$ and all $2 \leq i \leq \mu, z \in D_{M}$, or there exists $1 \leq k_{*} \leq m$ and $2 \leq i_{*} \leq \mu$ such that $\bar{L}_{k_{*}}\left(t_{i_{*}}(\bar{z} ; z)\right) \not \equiv 0$ on $M$. But this last possibility would readily contradict the minimality of $\mu$. Indeed, as the coefficients of the $\bar{L}_{k}$ 's are polynomial in $(z, \bar{z})$, all the terms $\bar{L}_{k_{*}}\left(t_{i}(\bar{z} ; z)\right)$ are still of the relatively rational form $\sum_{J} c_{i, J}(\bar{z}) z^{J} /\left(\sum_{J} d_{i, J}(\bar{z}) z^{J}\right)$ and after applying the CR derivation $\bar{L}_{k_{*}}$ to (4.17), and after chasing the denominators, we would obtain a similar polynomial relation

$$
\sum_{2 \leq i \leq \mu} g^{\alpha_{i}}(z) r_{i}^{\prime}(\bar{z} ; z) \equiv 0, \quad z \in M
$$

$r_{i}^{\prime}(\bar{z}, z)=\sum_{J} e_{i, J}(\bar{z}) z^{J}$, with a number $\mu_{1} \leq \mu-1$ of terms strictly less than $\mu$, and this relation is nontrivial, because $\bar{L}_{k_{*}}\left(t_{i_{*}}(\bar{z} ; z)\right) \not \equiv 0$, which yields a contradiction in this case. Thus, we are left to discuss the first possibility, where $\bar{L}_{k}\left(t_{i}(\bar{z} ; z)\right) \equiv 0$, for all $1 \leq k \leq m$ and all $2 \leq i \leq \mu, z \in D_{M}$. This case means that the real analytic $t_{i}(\bar{z} ; z)$ 's are smooth and CR over $D_{M}$. Hence they admit a holomorphic extension $s_{i}(z)$ to a neighborhood of $D_{M}$ in $\mathbb{C}^{n}$. The important Proposition 4.18 below shows that this holomorphic extension is in fact holomorphic algebraic. But then relation (4.17) gives

$$
g^{\alpha_{1}}(z)+\sum_{2 \leq i \leq \mu} s_{i}(z) g^{\alpha_{i}}(z) \equiv 0, \quad \text { for } z \in D_{M},
$$

which is a nontrivial algebraic relation between $\left(g_{1}, \ldots, g_{\kappa^{\prime}}\right)$, with

$$
\nabla^{\operatorname{tr}}\left(g_{1}, \ldots, g_{\kappa^{\prime}}\right)=\kappa^{\prime}
$$

this is again a contradiction. Granted Proposition 4.18 below, then Proposition 4.16 will be proved.

Proposition 4.18. - If $M$ is minimal at a Zariski-generic point and a relatively rational with respect to $z$ function

$$
t(\bar{z} ; z):=\sum_{J} a_{J}(\bar{z}) z^{J} /\left(\sum_{J} b_{J}(\bar{z}) z^{J}\right),
$$

where the coefficients $a_{J}(\bar{z})$ and $b_{J}(\bar{z})$ are holomorphic with respect to $\bar{z}$, is $C R$ on a Zariski open subset $D_{M}$ of $M$, then its holomorphic extension $s(z)$ to a neighborhood of $D_{M}$ in $\mathbb{C}^{n}$ is algebraic.

Proof. - We localize first at a minimal point of $D_{M}$. Now assuming the equation of $M$ is given by some local equations as in $\S 6.1$ below, we split the old

BULLETIN DE LA SOCiÉtÉ MATHÉMATIQUE DE FRANCE 
coordinates $z$ in the new coordinates $(w, z) \in \mathbb{C}^{m} \times \mathbb{C}^{d}$ and we complexify the equality

$$
s(w, \bar{z}+i \bar{\Theta}(w, \bar{w}, \bar{z})) \equiv \sum_{I, J} a_{I, J}(\bar{w}, \bar{z}) w^{I} z^{J} /\left(\sum_{I, J} b_{I, J}(\bar{w}, \bar{z}) w^{I} z^{J}\right),
$$

where each sum is finite, which is valuable for $(w, z) \in D_{M}$, to obtain

$$
\begin{aligned}
& s(w, \xi+i \bar{\Theta}(w, \zeta, \xi)) \\
& \quad \equiv\left[\sum_{I, J} a_{I, J}(\zeta, \xi) w^{I} z^{J} /\left(\sum_{I, J} b_{I, J}(\zeta, \xi) w^{I} z^{J}\right)\right]_{z:=\xi+i \bar{\Theta}(w, \zeta, \xi)}
\end{aligned}
$$

identically on the extrinsic complexification $\mathcal{M}$, i.e. identically as power series in $(w, \zeta, \xi)$. Of course, to complexify, we use the fact that

$$
M=\mathcal{M} \cap\{\zeta=\bar{w}, \xi=\bar{z}\}
$$

is maximally real in $\mathcal{M}$, hence a uniqueness set. Then (4.19) shows that $s$ is algebraic on each Segre surface $\mathcal{S}_{\zeta_{p}, \xi_{p}}$ and $\underline{\mathcal{S}}_{w_{p}, z_{p}}$ of $\mathcal{M}$, see (6.4) for their definition. We shall prove in Theorem 5.1 below that a holomorphic function defined in a neighborhood of a minimal CR-generic manifold is algebraic if and only if all its restrictions to Segre varieties are algebraic. Therefore $s$ is algebraic, as desired. Granted Theorem 5.1 below, this will complete the proof of Theorem 4.3 .

\section{Vector fields with complex algebraic flow and partial algebraicity of holomorphic mappings}

To conclude the proof of Theorem 4.3 above, it remains thus to establish the following statement about separate algebraicity of holomorphic mappings, which has been established by Sharipov-Sukhov (see [13]; see also [5] and $\S 10$ below) in the case where $M$ is Segre-transversal, instead of being minimal.

THEOREM 5.1. - Let $g \in \mathcal{H}\left(\mathcal{V}_{\mathbb{C}^{n}}(M), \mathbb{C}\right)$ be a holomorphic function, $M$ being a real algebraic CR-generic manifold which is minimal at a Zariski-generic point. Then $g$ is algebraic if and only if its restriction to each Segre variety of $M$ is algebraic.

After complexifying $M$ in a neighborhood of a minimal point, we shall be naturally led to deduce Theorem 5.1 from a more general statement. By slight abuse of terminology, we will call a Frobenius-integrable $k$-dimensional distribution $L$ over a complex manifold a ' $k$-vector field'. Such a distribution is said to have 'complex algebraic $k$-flow' if the local foliation that it induces (by Frobenius' theorem) coincides with a trivial product foliation by the $k$ dimensional polydiscs $\bigcup_{\theta} \Delta^{k} \times\{\theta\}$ in some local complex algebraic coordinate system. Its leaves will be called ' $k$-curves'. We refer the reader to $\S 7.1$ for further material about $\mathbb{L}$-orbits.

TOME $129-2001-\mathrm{N}^{\mathrm{O}} 4$ 
THEOREM 5.2. - Let $\mathbb{L}=\left\{L^{\alpha}\right\}_{\alpha \in A}$ be a system of $k_{\alpha}$-vector fields with complex algebraic flow on a small open connected set $U \subset \mathbb{C}^{n}$. Then

1) for $p \in U$, the $\mathbb{L}$-orbits $\mathcal{O}_{\mathbb{L}}(U, p)$ are complex algebraic manifolds;

2) a holomorphic function $g \in \mathcal{H}(U, \mathbb{C})$ is algebraic on each $\mathbb{L}$-orbit if and only if it is algebraic on each (complex algebraic) integral $k_{\alpha}$-curve of every element of $\mathbb{L}$.

In particular, if $\mathcal{O}_{\mathbb{L}}(U, p)$ contains an open subset of $\mathbb{C}^{n}$ for some $p \in U$, then a function $g \in \mathcal{H}(U, \mathbb{C})$ is algebraic on $U$ under the sole assumption that it is algebraic on $\mathbb{L}$-integral curves. This theorem, as well as its preliminary version given by Sharipov-Sukhov, generalizes the well known separate algebraicity principle in $\mathbb{C}^{n}$ proved in the book of Bochner-Martin [4]: A holomorphic function $g \in \mathcal{H}\left(\Delta^{n}, \mathbb{C}\right)$ is algebraic if and only if its restriction to every coordinate discs is algebraic. As a corollary to this Theorem 5.2, we shall also provide a new proof of the following theorem (cf. [2]).

TheOREM 5.3. - The CR orbits of a real algebraic CR manifold are algebraic.

We begin by explaining how Theorem 5.2 applies to provide a proof of Theorem 5.1. For this, we follow and summarize the constructions of [9].

\section{Foliations by complexified Segre varieties and algebraicity of CR-orbits of an algebraic CR manifold}

6.1. The extrinsic complexification of $M$. - Let $M \subset \mathbb{C}^{n}$ be a real algebraic CR-generic submanifold and set

$$
m:=\operatorname{dim}_{\mathrm{CR}} M, \quad d:=\operatorname{codim}_{\mathbb{R}} M, \quad m+d=n .
$$

Using the theory of algebraic functions, one can see that there exist holomorphic coordinates

$$
t=:(w, z) \in \mathbb{C}^{m} \times \mathbb{C}^{d}
$$

and a holomorphic algebraic $d$-vectorial function

$$
Q(\bar{w}, t)=\left(Q_{\ell}(\bar{w}, t)\right)_{1 \leq \ell \leq d}
$$

such that $M$ is given by the two equivalent systems of cartesian defining functions $z=\bar{Q}(w, \bar{t})$ or $\bar{z}=Q(\bar{w}, t)$. As $M$ is real, these two systems of equations are equivalent and there exists an invertible $d \times d$ matrix power series $a(t, \bar{t})$ such that

$$
z-\bar{Q}(w, \bar{t}) \equiv a(t, \bar{t})(\bar{z}-Q(\bar{w}, t)
$$

We can furthermore assume that $T_{0} M=\mathbb{C}_{w}^{m} \times \mathbb{R}_{x}^{d}$, in which case we shall write the equations of $M$ as follows:

$$
z=\bar{z}+i \bar{\Theta}(w, \bar{w}, \bar{z}) \quad \text { or } \quad \bar{z}=z-i \Theta(\bar{w}, w, z)
$$

BULLETIN DE LA SOCiÉtÉ MATHÉMATIQUE DE FRANCE 
where $\Theta$ vanishes to second order at the origin. In substance, such functions are locally holomorphic functions whose graph is contained in a complex algebraic set of the same dimension as the basis. Let now

$$
\tau:=(\bar{t})^{c}=:(\zeta, \xi) \in \mathbb{C}^{m} \times \mathbb{C}^{d}
$$

denote the complexification variable of $\bar{t}$. Then the extrinsic complexification of $M$ is given by the two, again equivalent, systems of cartesian defining equations $z=\bar{Q}(w, \tau)$ or $\xi=Q(\zeta, t)$. Following [9], we recall that there exist two systems of $m$-vector fields

$$
\mathcal{L}=\left(\mathcal{L}^{1}, \ldots, \mathcal{L}^{m}\right) \quad \text { and } \quad \underline{\mathcal{L}}=\left(\underline{\mathcal{L}}^{1}, \ldots, \underline{\mathcal{L}}^{m}\right),
$$

which are by definition the complexifications of two conjugate systems of $m$ vector fields spanning $T^{1,0} M$ and $T^{0,1} M$. These two systems $\mathcal{L}$ and $\underline{L}$ span two integrable subbundles of $T \mathcal{M}$ and they induce therefore two flow (Frobenius) foliations $\mathcal{F}_{\mathcal{L}}$ and $\mathcal{F}_{\underline{\mathcal{L}}}$ of $\mathcal{M}$, thanks to the integrability condition

$$
\left[\mathcal{L}^{i}, \mathcal{L}^{j}\right] \subset \mathcal{L} \quad \text { and } \quad\left[\underline{\mathcal{L}}^{i}, \underline{\mathcal{L}}^{j}\right] \subset \underline{\mathcal{L}} \text {. }
$$

As in [9], this fact can be rendered visible and straightforward just by writing in coordinates these two $m$-vector fields, using a vectorial and symbolic notation:

$$
\mathcal{M}: \quad \mathcal{L}=\frac{\partial}{\partial w}+\bar{Q}_{w}(w, \zeta, \xi) \frac{\partial}{\partial z} \quad \text { and } \quad \underline{\mathcal{L}}=\frac{\partial}{\partial \zeta}+Q_{\zeta}(\zeta, w, z) \frac{\partial}{\partial \xi} .
$$

Secondly, it is easy to observe that the two different (exercise) Segre varieties and conjugate Segre varieties as defined in [9], which can be rewritten as

$$
\left\{\begin{array}{l}
S_{\bar{t}_{p}}:=\left\{(w, z): z=\bar{z}_{p}+i \bar{\Theta}\left(w, \bar{w}_{p}, \bar{z}_{p}\right)\right\} \quad \text { and } \\
\bar{S}_{t_{p}}:=\left\{(\bar{w}, \bar{z}): \bar{z}=z_{p}-i \Theta\left(\bar{w}, w_{p}, z_{p}\right)\right\}
\end{array}\right.
$$

admit two different complexifications in $\mathcal{M}$, which we will denote by $\mathcal{S}_{\tau_{p}}$ and $\mathcal{S}_{t_{p}}$, and which can be written as follows:

$$
\left\{\begin{array}{l}
\mathcal{S}_{\tau_{p}}=\mathcal{S}_{\zeta_{p}, \xi_{p}}: \zeta=\zeta_{p}, \xi=\xi_{p}, z=\xi_{p}+i \bar{\Theta}\left(w, \zeta_{p}, \xi_{p}\right) \text { and } \\
\underline{\mathcal{S}}_{t_{p}}=\underline{\mathcal{S}}_{w_{p}, z_{p}}: w=w_{p}, z=z_{p}, \xi=z_{p}-i \Theta\left(\zeta, w_{p}, z_{p}\right) .
\end{array}\right.
$$

Here, the coordinates $t_{p}, \bar{t}_{p}$ or $\tau_{p}$ are thought to be fixed and the equations (6.3) define two $m$-dimensional complex submanifolds of $\mathcal{M}$ which coincide in fact with the coordinate intersections $\mathcal{M} \cap\left\{\tau=\tau_{p}\right\}$ and $\mathcal{M} \cap\left\{t=t_{p}\right\}$ respectively. Applying $\mathcal{L}$ to the equation of $\mathcal{S}_{\tau_{p}}$ and $\underline{\mathcal{L}}$ to the equations of $\underline{\mathcal{S}}_{t_{p}}$, we see that $\mathcal{L}$ is tangent to $\mathcal{S}_{\tau_{p}}$ and that $\underline{\mathcal{L}}$ is tangent to $\underline{\mathcal{S}}_{t_{p}}$. We can thus summarize these observations:

1) The $\mathcal{S}_{\tau_{p}}$ and the $\underline{\mathcal{S}}_{t_{p}}$ form families of integral complex algebraic manifolds for $\mathcal{L}$ and $\underline{\mathcal{L}}$ respectively.

2) The $\mathcal{S}_{\tau_{p}}$ are leaves of the flow foliation $\mathcal{F}_{\mathcal{L}}$ of $\mathcal{M}$ by $\mathcal{L}$ and the $\underline{\mathcal{S}}_{t_{p}}$ are leaves of the flow foliation $\mathcal{F}_{\underline{\mathcal{L}}}$ of $\mathcal{M}$ by $\underline{\mathcal{L}}$.

TOME $129-2001-\mathrm{N}^{\mathrm{O}} 4$ 
3) Therefore, the two $m$-flows of $\mathcal{L}$ and of $\underline{\mathcal{L}}$ are both complex algebraic, because the families $\mathcal{F}_{\mathcal{L}}=\bigcup_{\tau_{p} \in \mathbb{C}^{n},\left|\tau_{p}\right|<\delta} \mathcal{S}_{\tau_{p}}$ and $\mathcal{F}_{\underline{\mathcal{L}}}=\bigcup_{t_{p} \in \mathbb{C}^{n},\left|t_{p}\right|<\delta} \underline{\mathcal{S}}_{t_{p}}$ are clearly tangentially and transversally algebraic.

Finally, we will need the following straightforward lemma about complexifications of Lie algebras including a well known characterization of finite type (orbit-minimality) at a point, valuable in the real analytic and real algebraic categories (see also [9]). Let $p^{c}:=(p, \bar{p}) \in \mathcal{M}$ be the complexification of the point $p$. Then:

LEMma 6.5. - The following five properties are equivalent:

(a) $\operatorname{Lie}_{p}\left(T^{1,0} M, T^{0,1} M\right)=\mathbb{C} \otimes T_{p} M$;

(b) $\operatorname{Lie}_{p}\left(T^{c} M\right)=T_{p} M$;

(c) $(M, p)$ is $T^{c} M$-orbit-minimal;

(d) $\operatorname{Lie}_{p^{c}}(\mathcal{L}, \mathcal{L})=T_{p^{c}} \mathcal{M}$

(e) $\left(\mathcal{M}, p^{c}\right)$ is $\{\mathcal{L}, \underline{\mathcal{L}}\}$-orbit-minimal.

6.6. Deduction of Theorem 5.1. - As announced in $\S 5$, Theorem 5.1 can be therefore deduced from the more general statement Theorem 5.2, thanks to the following two facts. Let there be given a holomorphic function $g \in \mathcal{H}\left(\mathcal{V}_{\mathbb{C}^{n}}\left(p_{0}\right), \mathbb{C}\right)$ defined in a neighborhood of a minimal point $p_{0}$ of $M$, which we think to be the origin in the above coordinates $(w, z)$ for $M$. Then:

1) This function $g$ induces a function $g^{c}: \mathcal{M} \rightarrow \mathbb{C}$ defined by

$$
g^{c}(w, z, \zeta, \xi):=g(w, z) .
$$

Since by assumption $g^{c}$ is algebraic on every Segre variety $S_{\bar{t}_{p}}$, then $g^{c}$ is algebraic on every complexified Segre variety $\mathcal{S}_{\tau_{p}}$. On the other hand, $g^{c}$ is also clearly algebraic on every conjugate complexified Segre variety $\underline{\mathcal{S}}_{t_{p}}$, because it is then constant equal to $g\left(w_{p}, z_{p}\right)$.

2) According to Lemma $6.5, \mathcal{M}$ is orbit-minimal at $\left(p_{0}, \bar{p}_{0}\right)$ for the system $\mathbb{L}:=\{\mathcal{L}, \underline{\mathcal{L}}\}$ composed of (only) two $m$-vector fields. Because by step 1 ) above, $g^{c}$ is algebraic on each $m$-integral manifold of this system, then Theorem 5.2 clearly yields the algebraicity of $g^{c}$ over $\mathcal{M}$, whence $g$ is algebraic.

6.7. Deduction of Theorem 5.3. - Theorem 5.3 can also be deduced from Theorem 5.2, because of the following relation between the CR-orbits $\mathcal{O}_{\mathrm{CR}}(M, p)=\mathcal{O}_{L, \bar{L}}(M, p)$ of points $p$ in $M$ and the $\{\mathcal{L}, \underline{\mathcal{L}}\}$-orbits $\mathcal{O}_{\mathcal{L}, \underline{\mathcal{L}}}\left(\mathcal{M}, p^{c}\right)$ of points $p^{c} \in \mathcal{M}$, which is established in [9]. Let $\pi_{t}:(t, \tau) \mapsto t$ denote the projection on the first coordinate space, which is defined over the complexification space $\mathbb{C}_{t}^{n} \times \mathbb{C}_{\tau}^{n}$. Let $\underline{\Lambda}:=\{(t, \tau): \tau=\bar{t}\}$ denote the antidiagonal.

Proposition 6.8 (see [9]). - We have the following reciprocal complexification relations: 
1) $\mathcal{O}_{\mathrm{CR}}(M, p)^{c}=\mathcal{O}_{\mathcal{L}, \underline{\mathcal{L}}}\left(\mathcal{M}, p^{c}\right)$

2) $\mathcal{O}_{\mathrm{CR}}(M, p)=\pi_{t}\left(\underline{\Lambda} \cap \mathcal{O}_{\mathcal{L}, \underline{\mathcal{L}}}\left(\mathcal{M}, p^{c}\right)\right)$.

Granted this proposition and thanks to the observation that the the $m$-flows of $\mathcal{L}$ and of $\underline{\mathcal{L}}$ are naturally algebraic, it now follows from Theorem 5.2,1), that the orbits $\mathcal{O}_{\mathcal{L}, \mathcal{L}}(\mathcal{M}, q)$ of various points $q \in \mathcal{M}$ are all algebraic, whence with $q:=p^{c}$, we deduce from 2) above that the CR-orbits $\mathcal{O}_{\mathrm{CR}}(M, p)$ are all real algebraic. To conclude the proof of Theorem 2.5, it now only remains to establish Theorem 5.2.

\section{Algebraicity of orbits of vector fields having algebraic flow and local holomorphic mappings of nowhere minimal CR-generic manifolds}

7.1. Definition of the concatenated flow maps. - As in Theorem 5.2, let $\mathbb{L}=\left\{L^{\alpha}\right\}_{\alpha \in A}$ be a finite set of nonzero holomorphic $k_{\alpha}$-vector fields defined on an open connected set $U \subset \mathbb{C}^{n}$. For the moment's discussion, we do not assume algebraicity of their flows. As all our reasonings will be local, we shall assume that $U=\Delta^{n}$. Of course, after demultiplying any such $k_{\alpha}$-vector in some $k_{\alpha}$ linearly independent 1-vector fields, we can also assume that all the $L^{\alpha}$ 's are (usual) 1-vector fields. Thus, denote these vector fields by

$$
L^{\alpha}=\sum_{j=1}^{n} a_{j \alpha} \frac{\partial}{\partial z_{j}},
$$

where $a_{j \alpha}(z) \in \mathcal{H}\left(\Delta^{n}\right)$. By a well known theorem, the global flow of each such vector field $L^{\alpha} \in \mathbb{L}$, say

$$
\phi_{\alpha}:(t, p) \longrightarrow \exp \left(t L^{\alpha}\right)(p) \in \Delta^{n},
$$

is defined over a certain maximal subdomain $\Omega_{\alpha}$ of $\mathbb{C} \times \Delta^{n}$ which contain $\Delta^{n} \times\{0\}$ and this global flow is a holomorphic map over that subdomain. However, following our version of Sussmann's constructions elaborated in [9] in the (paradigmatic) analytic context, we shall proceed as follows in order not to deal with these domains of the global flows $\phi_{\alpha}$ 's. Let us now denote by $(t, p) \mapsto L_{t}^{\alpha}(p)$ these flow maps in the sequel.

To define $\mathbb{L}$-orbits, we shall need to consider concatenated flow maps of the form $L_{t_{k}}^{\alpha_{k}} \circ \cdots \circ L_{t_{1}}^{\alpha_{1}}(p)$. By definition, the $\mathbb{L}$-orbit $\mathcal{O}_{\mathbb{L}}\left(\Delta^{n}, p\right)$ of a point $p \in \Delta^{n}$ is just the set of all such elements $L_{t_{k}}^{\alpha_{k}} \circ \cdots \circ L_{t_{1}}^{\alpha_{1}}(p) \in \Delta^{n}$ where $\alpha_{1}, \ldots, \alpha_{k} \in A$ and where $k \in \mathbb{N}_{*}$ is a priori unbounded. Nevertheless, after comparison with the (semi-global) constructions in [16], it appears that, due to the fact that the $L^{\alpha}$ 's are defined over $\Delta^{n}$, this set of points gives nothing more interesting for $k>3 n$. According to Nagano's theorem (revisited by Sussmann with the above definitions), the main property of $\mathbb{L}$-orbits lies in the fact that they are closed complex analytic submanifolds of $\Delta^{n}$ passing through $p$ and

TOME $129-2001-\mathrm{N}^{\mathrm{O}} 4$ 
for this, $k \leq 3 n$ suffices. Furthermore, there is another phenomenon which is due to the principle of analytic continuation and which is quite well known (cf. [16]): the local and the global CR-orbits coincide locally in the real analytic category, which is false in the smooth $\mathcal{C}^{\infty}$ category. Therefore, to study orbits and to fix the domains of the concatenated flow maps, it suffices to choose some positive number $\delta>0$ such that for all $p \in 2^{-n} \Delta^{n}$, all $k \in \mathbb{N}_{*}$ with $k \leq 3 n$ and all $t_{1}, \ldots, t_{k}$ with $\left|t_{j}\right| \leq \delta$, one has $L_{t_{k}}^{\alpha_{k}} \circ \cdots \circ L_{t_{1}}^{\alpha_{1}}(p) \in \Delta^{n}$, which is clearly possible by continuity of the flow maps. In summary, there is no restriction to define from the beginning the $\mathbb{L}$-orbits of various points $p \in 2^{-n} \Delta^{n}$ as the set of all elements $L_{t_{k}}^{\alpha_{k}} \circ \cdots \circ L_{t_{1}}^{\alpha_{1}}(p) \in \Delta^{n}$ where $\alpha_{1}, \ldots, \alpha_{k} \in A$, where $k \leq 3 n$ and where $\left|t_{j}\right| \leq \delta$. We shall say that the point $p$ is $\mathbb{L}$-orbit-minimal if its $\mathbb{L}$-orbit (with this definition) contains an open neighborhood of $p$ in $\Delta^{n}$.

7.2. Algebraicity of the $\mathbb{L}$-orbits. - Assuming now that all the flows of the elements of $\mathbb{L}$ are complex algebraic, we can easily check part 1) of Theorem 5.2. Indeed, according to the construction given in [9], for an arbitrary fixed point $p \in 2^{-n} \Delta^{n}$, there exists a integer $e_{p} \leq n$ such that

1) the holomorphic maps $\left(t_{1}, \ldots, t_{k}\right) \mapsto L_{t_{k}}^{\alpha_{k}} \circ \cdots \circ L_{t_{1}}^{\alpha_{1}}(p) \in \Delta^{n}$ are of generic rank equal to $e_{p}$ for all $e_{p} \leq k \leq 3 n$;

2) there exist $t_{1}^{*}, \ldots, t_{e_{p}}^{*} \in \mathbb{C}$ arbitrarily close to 0 such that the map

$$
\Gamma_{e_{p}}:\left(t_{1}, \ldots, t_{e_{p}}\right) \longmapsto L_{-t_{1}^{*}}^{\alpha_{1}} \circ \cdots \circ L_{-t_{e_{p}}^{*}}^{\alpha_{e_{p}}} \circ L_{t_{e_{p}}}^{\alpha_{e_{p}}} \circ \cdots \circ L_{t_{1}}^{\alpha_{1}}(p) \in \Delta^{n}
$$

is of constant rank $e_{p}$ over a neighborhood $\mathcal{T}^{*}$ of $\left(t_{1}^{*}, \ldots, t_{e_{p}}^{*}\right)$ in $(\delta \Delta)^{e_{p}}$.

3) The map $\Gamma_{e_{p}}$ (clearly) satisfies $\Gamma_{e_{p}}\left(t_{1}^{*}, \ldots, t_{e_{p}}^{*}\right)=p$.

4) The image $\Gamma_{e_{p}}\left(\mathcal{T}^{*}\right)$ is an $e_{p}$-dimensional submanifold of $\Delta^{n}$ through $p$ which coincides with the $\mathbb{L}$-orbit $\mathcal{O}_{\mathbb{L}}\left(\Delta^{n}, p\right)$ of $p$ in a neighborhood of $p$ in $\Delta^{n}$

Notice that this statement clearly shows that the $\mathbb{L}$-orbit of $p$ is a submanifold and that it provides this orbit with the regularity of the concatenated flow $\operatorname{map} \Gamma_{e_{p}}$.

End of proof of Theorem 5.2. - As we supposed that the flows are complex algebraic, the above maps $\Gamma_{e_{p}}$ are algebraic and then clearly the orbits are complex algebraic by 4), q.e.d. Of course, similar other regularity properties of orbits in other differentiable categories rely upon the regularity $\mathcal{C}^{\infty}, \mathcal{C}^{k}, \mathcal{C}^{l, \alpha}$ of the flow maps. This proves part 1) of Theorem 5.2 and we shall establish part 2) in $\S 8$ below.

7.4. Flow-bow theorem. - Now, as in the differentiable theory, it is easy to deduce from the above four properties 1), 2), 3), 4) a complex algebraic flow-box theorem, that will be useful. 
THEOREM 7.5. - Let $p \in U$ and set $e_{p}=\operatorname{dim} \mathcal{O}_{\mathbb{L}}\left(\Delta^{n}, p\right)$. Then there exist a neighborhood $V$ of $p$ in $\Delta^{n}$ and a biholomorphism $\Phi: V \rightarrow \Delta^{e_{p}} \times \Delta^{n-e_{p}}$ with complex algebraic components such that

1) $\Phi^{-1}\left(\Delta^{e_{p}} \times\{0\}\right)=\mathcal{O}_{\mathbb{L}}\left(\Delta^{n}, p\right) \cap V$;

2) for all $\zeta_{\left(n-e_{p}\right)} \in \Delta^{n-e_{p}}, \Phi^{-1}\left(\Delta^{e_{p}} \times\left\{\zeta_{\left(n-e_{p}\right)}\right\}\right)$ is contained in a single $\mathbb{L}$-orbit;

3) The function $\Delta^{n} \ni p \mapsto \operatorname{dim}_{\mathbb{C}} \mathcal{O}_{\mathbb{L}}\left(\Delta^{n}, p\right) \in \mathbb{N}$ is upper semicontinuous.

As usual, we can now derive from Theorem 7.5 a complex algebraic Frobenius theorem. As the dimension of orbits is an integer depending upper-semicontinuously on the point $p$, it assumes its maximal value $:=e$ at a Zariskigeneric point $p \in \Delta^{n}$. Let $\mathcal{V}_{M}(p)$ be the intersection of a small polydisc $\mathcal{V}_{\mathbb{C}^{n}}(p)$ with $M$.

Corollary 7.6. - Let $p \in \Delta^{n}$ with

$$
\operatorname{dim}_{\mathbb{C}} \mathcal{O}_{\mathbb{L}}\left(\Delta^{n}, p\right)=\max _{q \in \mathcal{V}_{\Delta^{n}}(p)} \operatorname{dim}_{\mathbb{C}} \mathcal{O}_{\mathbb{L}}\left(\Delta^{n}, q\right)=: e .
$$

Then $\mathcal{V}_{\Delta^{n}}(p)$ is algebraically foliated by $\mathbb{L}$-integral manifolds of dimension e.

Using the properties summarized in (7.3), it is also easy to show that there exists a proper algebraic subvariety of $\Delta^{n}$ outside of which the dimension of $\mathbb{L}$-orbits is maximal equal to $e$. Finally, we can derive from the previous considerations a real algebraic CR-foliation theorem, useful to prove Theorem 2.7.

Corollary 7.7. - Suppose that $p \in M$ is a point at which the dimension of nearby $C R$ orbits is maximal and locally constant equal to $2 m+d-e$. Then a neighborhood $\mathcal{V}_{M}(p)$ is real algebraically foliated by CR-orbits and there exist e holomorphic algebraic functions $h_{1}, \ldots, h_{e}$ with $\partial h_{1} \wedge \cdots \wedge \partial h_{e}(p) \neq 0$ such that

1) $M$ is contained in $\left\{h_{1}=\bar{h}_{1}, \ldots, h_{e}=\bar{h}_{e}\right\}$. In other words, $M$ is contained in a transverse intersection of e Levi-flat hypersurfaces in general position;

2) each manifold $M_{c}=M \cap\left\{h_{1}=c_{1}, \ldots, h_{e}=c_{e}\right\}$ is a CR-orbit of $M$.

Proof. - As in the proof of Proposition 6.82 ), we can intersect the foliation provided by Corollary 7.6 with the antidiagonal $\underline{\Lambda}$ to produce the algebraic foliation of $M$ by its CR orbits. Let therefore $\bar{h}_{1}, \ldots, h_{e}$ be real algebraic functions over $(M, p)$ with linearly independent differential such that the levelsets $\left\{h_{1}=c_{1}, \ldots, h_{e}=c_{e}\right\}, c_{j} \in \mathbb{R}$, are the plaques of this foliation. Since the $h_{j}$ 's are constant on CR-orbits, they are CR on $M$. Consequently, they extend as holomorphic algebraic functions in a neighborhood of $(M, p)$ in $\mathbb{C}^{n}$, by the theorem of Severi-Tomassini.

TOME $129-2001-\mathrm{N}^{\mathrm{O}} 4$ 
Granted Corollary 7.7, a nowhere minimal CR-generic $M$ is contained in at least one Levi-flat algebraic hypersurface in a neighborhood of a Zariski-generic point. We are now in position to prove Theorem 2.7.

TheOREM 7.8. - Let $M$ be a real algebraic CR-generic manifold in $\mathbb{C}^{n}$, let $p \in M$, assume that $(M, p)$ is contained in the Levi-flat hypersurface $\left\{z_{n}=\bar{z}_{n}\right\}$ and that $M$ possesses a nontrivial infinitesimal $C R$-automorphism inducing an algebraic foliation. Then $M$ admits a local one parameter family of nonalgebraic biholomorphic self-maps.

Proof. - Let $\mathcal{X}$ be a $(1,0)$-vector field with holomorphic algebraic coefficients in a neighborhood of $p$ and let $\mathcal{K}:=\operatorname{Re}(\mathcal{X})$ be the associated infinitesimal CRautomorphism, which is tangent to $(M, p)$. Since by assumption, the foliation induced by $\mathcal{X}$ is complex algebraic, we can assume after perharps multiplying $\mathcal{X}$ by a nonzero algebraic function that its complex flow $(u, z) \mapsto \exp (u \mathcal{X})(z)=$ : $\varphi(u, z)$ is algebraic, see Lemma 9.12 below. It is well known that for $u$ real, the flow of $\mathcal{X}$ coincides with the real flow of $\mathcal{K}=\operatorname{Re}(\mathcal{X})$, hence it stabilizes $(M, p)$. By definition,

$$
\partial_{u} \varphi(u, z)=\mathcal{X}(\varphi(u, z)) .
$$

Since $\mathcal{X} \neq 0$, then obviously $\partial_{u} \varphi(u, z) \not \equiv 0$. Let now $\varpi\left(z_{n}\right)$ be an arbitrary nonzero holomorphic nonalgebraic function with $\varpi(0)=0$ which is real, i.e. $\bar{\varpi}\left(z_{n}\right) \equiv \varpi\left(z_{n}\right)$ and satisfies $\partial_{u} \varphi\left(\varpi\left(z_{n}\right), z\right) \not \equiv 0$. Then the map

$$
z \longmapsto \exp \left(\varpi\left(z_{n}\right) \mathcal{X}\right)(z)=\varphi\left(\varpi\left(z_{n}\right), z\right)
$$

is a holomorphic nonalgebraic biholomorphism, because, if it where algebraic, the two conditions: $\varphi\left(\varpi\left(z_{n}\right), z\right)$ is algebraic and $\partial_{u} \varphi\left(\varpi\left(z_{n}\right), z\right) \not \equiv 0$ would clearly imply that $\varpi$ is algebraic. Finally, this map sends $M$ into $M$, because $\operatorname{Re}(\mathcal{X})$ is an infinitesimal CR-automorphism of $M$ and because $\varpi$ is real on $M$, which is the crucial point. To get a one-parameter family of such maps, just take $\varphi\left(s \varpi\left(z_{n}\right), z\right)$ with $s \in \mathbb{R}$ small.

\section{Partial algebraicity}

8.1. Propagation of algebraicity. - Let $\mathbb{L}=\left\{L^{\alpha}\right\}_{\alpha \in A}$ be a set of vector fields defined over $\Delta^{n}$ in $\mathbb{C}^{n}$ with complex algebraic flow. Without loss of generality, we can assume that $k_{\alpha}=1$ for all $\alpha \in A$. Following our scheme of proof for Theorem 2.5, it remains now only to prove Theorem 5.2, 2), namely:

THEOREM 8.2. - A holomorphic function $g \in \mathcal{H}\left(\Delta^{n}, \mathbb{C}\right)$ is algebraic on each $\mathbb{L}$-orbit if and only it is algebraic on each $\mathbb{L}$-integral curve. 
Proof. - We can assume $\mathbb{L} \neq\{0\}$. Of course, any small open piece of an $\mathbb{L}$-orbit being algebraically locally equivalent to some $\Delta^{e}$ by part 1 ) of Theorem 5.2, part 2) is then reduced to the case where $U$ is a single $\mathbb{L}$-orbit, i.e. $U$ is $\mathbb{L}$-minimal.

TheOrem 8.2'. - Assume that $\Delta^{n}$ is $\mathbb{L}$-minimal at 0 and let $g \in \mathcal{H}\left(\Delta^{n}, \mathbb{C}\right)$. If $g$ is algebraic on each $\mathbb{L}$-integral curve, then $g$ is algebraic over $\Delta^{n}$.

REMARK. - This theorem is proved by Sharipov-Sukhov in [13] in case there exist $L^{1}, \ldots, L^{n} \in \mathbb{L}$ and $p \in U$ such that $\operatorname{rk}_{\mathbb{C}}\left(L^{1}(p), \ldots, L^{n}(p)\right)=n$, an assumption which corresponds essentially to Segre-transversality of $M[5]$ in $\mathbb{C}^{n}$ in place of the more general assumption of $\{\mathcal{L}, \underline{\mathcal{L}}\}$-orbit minimality in the complexification $\mathcal{M}$.

We denote by $U$ a neighborhood of 0 in $\Delta^{n}$ such that at every point $q \in U$, the local $\mathbb{L}$-orbit of $q$ in $U$ contains an open neighborhood of $q$. We shall say that $U$ is $\mathbb{L}$-minimal (locally). Hence for each germ $\Lambda \subset U$ of a $\mathbb{C}$-algebraic manifold with $\operatorname{dim} \Lambda<n$ and $\Lambda \neq \varnothing$, there exist $p \in \Lambda$ and $L \in \mathbb{L}$ such that $L(p) \notin T_{p} \Lambda$. Otherwise, $\Lambda$ would be $\mathbb{L}$-integral, in contradiction with $\mathbb{L}$ minimality of $U$. Of course, $\left\{q \in \Lambda: L(q) \in T_{q} \Lambda, \forall L \in \mathbb{L}\right\}$ is a proper closed $\mathbb{C}$-algebraic subset of $\Lambda$. More generally, we shall need to consider this nontangentiality property along some families of such manifolds $\Lambda$ which foliate subdomains of $\Delta^{n}$.

8.3. Complex algebraic foliations. - A regular holomorphic foliation $\mathcal{F}$ of a subdomain $V \subset \mathbb{C}^{n}$ is called algebraic if its transition maps are $\mathbb{C}$-algebraic. For short, we shall say in the sequel that ' $\mathcal{F}$ is an $\mathcal{A}$-foliation'. We denote by $\mathcal{A}(X, \mathbb{C})$ the ring of holomorphic algebraic functions on the complex manifold $X$. Let now $\Lambda_{\mathcal{F}}(p)$ be the leaf of $\mathcal{F}$ through $p$ and let $m:=\operatorname{dim}_{\mathbb{C}} \mathcal{F}$. We will consider only local foliations, so that there is no restriction to assume that all neighborhoods $\mathcal{V}_{\mathbb{C}^{n}}(q), V, W$, etc. in the sequel are foliation boxes, which will simplify our considerations. Recall that this means that $\mathcal{F}$ is represented in an open set, say $V$, by an algebraic (global) coordinate system $\Phi: V \rightarrow \Delta^{m} \times \Delta^{m-n}$, with respect to which the leaves of $\mathcal{F}$ in $\Delta^{n}$ are represented by the 'plaques' $\Delta^{m} \times\left\{\zeta_{(n-m)}\right\}$. Thus, in $V$, the leaves of $\Lambda_{\mathcal{F}}(q)$ of the $\mathcal{A}$-foliation $\mathcal{F}$ are simply the preimages $\Phi^{-1}\left(\Delta^{m} \times\left\{\zeta_{(n-m)}(q)\right\}\right)$, if we denote

$$
\Phi(q)=\zeta_{(m)}(q) \times \zeta_{(n-m)}(q) .
$$

Let now $p \in V$, and choose an arbitrary complex algebraic $(n-m)$-dimensional submanifold $H \subset V$ passing through $p$ and satisfying $T_{p} H \oplus T_{p} \Lambda_{\mathcal{F}}(p)=T_{p} V$. Thus $\operatorname{dim}_{\mathbb{C}} H+\operatorname{dim}_{\mathbb{C}} \mathcal{F}=n$. Then there exists a neighborhood $W:=\mathcal{V}_{\mathbb{C}^{n}}(p)$ such that $\bigcup_{q \in H} \Lambda_{\mathcal{F}}(q) \cap W=\mathcal{F}_{\mid W}$. Again, $W$ is a foliation box here and the leaves $\Lambda_{\mathcal{F}}(q)$ are embedded closed $\mathbb{C}$-algebraic submanifolds of $W$ which are the plaques of $\left.\mathcal{F}\right|_{W}$. Finally, recall that to any vector field $L \in \mathbb{L}$ with $L \neq 0$

TOME $129-2001-\mathrm{N}^{\mathrm{O}} 4$ 
and to any point $p \in U$ with $L(p) \neq 0$, there is associated a neighborhood $V=\mathcal{V}_{\mathbb{C}^{n}}(p)$ which is algebraically foliated by $L$-integral curves. Such foliations will be denoted by $\mathcal{F}_{\ell}$ in the sequel.

8.4. Description of the proof of Theorem 8.2' - We now introduce an important notation. We shall write $g \in \mathcal{A}_{\mathcal{F}}(V)$ if $g$ restricted to each leaf of $\mathcal{F}_{\mid V}$ is algebraic. To establish Theorem $8.2^{\prime}$, we will prove the following statement by induction on the integer $m \in \mathbb{N}$ :

(*) For all $m, 1 \leq m \leq n$, there exist $p \in U$ and an $m$-dimensional foliation $\mathcal{F}$ of $V=\mathcal{V}_{\mathbb{C}^{n}}(p)$ such that $g \in \mathcal{A}_{\mathcal{F}}(V)$.

Then the desired Theorem $8.2^{\prime}$ will be just (*) for $m=n$. We already know (*) for $m=1$, since $g \in \mathcal{A}_{\mathcal{F}}\left(\mathcal{F}_{L}\right)$ for every nonzero vector field $L \in \mathbb{L}$. Let $m \leq n-1$. We have to assume $(*)$ for $m$, i.e. $\left({ }^{*}\right)_{m}$ and to deduce $(*)$ for $m+1$, i.e. $\left({ }^{*}\right)_{m+1}$. We can describe now this inductional implication in the large, except some two main lemmas that will be rejected below. First, by $\mathbb{L}$-minimality of $U$, we have obviously:

Lemma 8.5. - Let $p \in U, V=\mathcal{V}_{\mathbb{C}^{n}}(p)$, and let $\mathcal{F}$ be an $\mathcal{A}$-foliation of $V$ with $\operatorname{dim}_{\mathbb{C}} \mathcal{F}=m, 1 \leq m \leq n-1$. Then for all $q \in V$, there exist $r \in \Lambda_{\mathcal{F}}(q)$ arbitrarily close to $q$ and $L \in \mathbb{L}$ such that the vector $L(r)$ is not tangent to the leaf $\Lambda_{\mathcal{F}}(q)$.

Indeed, otherwise, the manifold $\Lambda_{\mathcal{F}}(q)$ would be an $\mathbb{L}$-integral manifold in a neighborhood of $q$ of positive codimension, a contradiction with local $\mathbb{L}$-minimality of $U$ at every point. Let now $z=\left(z_{1}, \ldots, z_{n}\right)$ denote algebraic coordinates on $U$. Here is our first main lemma, inspired from [13], which will be the first step of our proof of the inductional implication. This lemma will state that if $g$ is algebraic on the leaves of $\mathcal{F}_{\mid V}$, then $g$ and all its derivatives are algebraic on the leaves of $\mathcal{F}_{\mid W}$ after restriction to a possibly smaller subdomain $W \subset V$.

LEMMA 8.6. - If $g \in \mathcal{A}_{\mathcal{F}}(V)$, then there exists a nonempty subdomain $W \subset V$ such that all partial derivatives $\partial_{z}^{\beta} g \in \mathcal{A}_{\mathcal{F}}(W)$ too, for all $\beta \in \mathbb{N}^{n}$.

It is easy to check that this condition is independent of coordinates.

LEMMA 8.7. - Let $V \subset \mathbb{C}^{n}$ be a domain, let $z$ be complex algebraic coordinates on $V$, let $\mathcal{F}$ be an $\mathcal{A}$-foliation on $V$, let $g \in \mathcal{H}(V, \mathbb{C})$ and let $\Phi: V \rightarrow \Phi(V)$ be an $\mathcal{A}$-biholomorphism, $w=\Phi(z)$. If $\partial_{z}^{\beta} g \in \mathcal{A}_{\mathcal{F}}(V)$ for all $\beta \in \mathbb{N}^{n}$, then $\partial_{w}^{\beta}\left(g \circ \Phi^{-1}\right) \in \mathcal{A}_{\Phi_{*}} \mathcal{F}(\Phi(V))$, for all $\beta \in \mathbb{N}^{n}$.

Proof. - Simple application of the chain rule, because there exist universal polynomials $P_{\beta}$ with $\partial_{w}^{\beta}\left(g \circ \Phi^{-1}\right)=P_{\beta}\left(\left\{\partial_{w}^{\gamma} \Phi^{-1}\right\}_{\gamma \leq \beta},\left\{\left(\partial_{z}^{\gamma} g\right) \circ \Phi^{-1}\right\}_{\gamma \leq \beta}\right)$.

To achieve the second step of the proof, we have to construct a foliation $\mathcal{F}^{1+}$ with $\operatorname{dim} \mathcal{F}^{1+}=m+1$ satisfying $\left({ }^{*}\right)_{m+1}$. Let $p, V$ and $\mathcal{F}$ be as in $\left({ }^{*}\right)_{m}$ and let $q \in V$ be arbitrary with $V$ like the $W$ in Lemma 8.6 (shrinking $V$ if necessary). 
Choose now $r \in \Lambda_{\mathcal{F}}(q)$ as in Lemma 8.5 with $L(r) \notin T_{r} \Lambda_{\mathcal{F}}(q)$. Changing notation, we will now denote this $r$ by $p$. Further, let $H$ be a piece of an $\mathcal{A}$-manifold through $p$ with $T_{p} H \oplus T_{p} \Lambda_{\mathcal{F}}(p)=T_{p} V$, so $\operatorname{dim}_{\mathbb{C}} H=n-m$. Then $\mathcal{F}_{\mid V}=$ $\bigcup_{q \in H} \Lambda_{\mathcal{F}}(q) \cap V$ (after shrinking $V$ and still with the convention about small open sets being foliation boxes), where, of course, $\Lambda_{\mathcal{F}}(q) \cap \Lambda_{\mathcal{F}}\left(q^{\prime}\right)=\varnothing$ if $q \neq q^{\prime}$. Now, instead of $H$, let us choose an arbitrary piece $H^{1+}$ of an $\mathcal{A}$-manifold with $p \in H^{1+}$ and

$$
T_{p} H^{1+} \oplus \mathbb{C} L(p) \oplus T_{p} \Lambda_{\mathcal{F}}(p)=T_{p} V
$$

so $\operatorname{dim}_{\mathbb{C}} H^{1+}=n-m-1$. To the triple $\left(\mathcal{F}, L, H^{1+}\right)$, we can finally associate an $\mathcal{A}$-foliation $\mathcal{F}^{1+}=\mathcal{F}^{1+}\left(\mathcal{F}, L, H^{1+}\right)$ of $\mathcal{V}_{\mathbb{C}^{n}}(p)$ with $\operatorname{dim}_{\mathbb{C}} \mathcal{F}^{1+}=m+1$, which is defined as follows and will be the important object to get $\left({ }^{*}\right)_{m+1}$. Simply, this foliation will be constructed by flowing the leaves of $\mathcal{F}$ a bit along the nontangential flow lines of $L$, thus gaining one unit in dimension. Precisely:

1) the leaves $\Lambda_{\mathcal{F}^{1+}}(q):=\left\{L_{s}(r):|s|<\delta, r \in \Lambda_{\mathcal{F}}(q)\right\}$, for $q \in H^{1+}$;

2) $\mathcal{F}^{1+}=\bigcup_{q \in H^{1+}} \Lambda_{\mathcal{F}^{1+}}(q)$.

By construction, the foliation $\mathcal{F}^{1+}$ clearly has the following structure:

3) $\mathcal{F}_{L}$ is a subfoliation of $\mathcal{F}^{1+}$ in $\mathcal{V}_{\mathbb{C}^{n}}(p)$;

4) $\mathcal{F}$ is not in general a subfoliation of $\mathcal{F}^{1+}$, but each leaf of $\mathcal{F}^{1+}$ contains at least one leaf of $\mathcal{F}: \Lambda_{\mathcal{F}^{1+}}(q) \supset \Lambda_{\mathcal{F}}(q)$ for $q \in H^{1+}$.

IMPORTANT REMARK. - Such a step-by-step construction of several foliations is necessary because, even if there may exist $n$ linearly independent vector fields $L^{1}, \ldots, L^{n}$ in $\mathbb{L}$ over $U$, there might not exist a coordinate system whose coordinate line correspond to the flow lines of the $L^{j}$ 's. More specifically, and for the same reason, even in the above construction of $\mathcal{F}^{1+}$, there does not exist in general a system of coordinates $\left(x_{1}, \ldots, x_{m}, x_{m+1}, y_{1}, \ldots, y_{n-m-1}\right)$ such that the $x_{m+1}$-lines correspond to the integral curves of $L$ and such that, simultaneously, the $\left(x_{1}, \ldots, x_{m}\right)$-planes correspond to leaves of $\mathcal{F}$. In particular, one cannot in fact reduce our Theorem $8.2^{\prime}$ directly to a classical theorem due to Bochner-Martin (1949) [3] which states that given $g \in \mathcal{H}\left(\Delta^{n}, \mathbb{C}\right)$ algebraic on $z_{i}$-lines, $i=1, \ldots, n$, then $g$ is algebraic. To argue this general impossibility, let us consider for instance for $n=2$, some two linearly independent vector fields

$$
L_{1}:=\frac{\partial}{\partial x} \quad \text { and } \quad L_{2}:=\frac{\partial}{\partial y}+a(x, y) \frac{\partial}{\partial x}
$$

(general form of such a pair of vector field after a convenient choice of coordinates $(x, y))$. In this case, it is easy to see that there further exists a biholomorphism of $\mathcal{V}_{\mathbb{C}^{2}}(0)$ transforming $L_{1}$ and a multiple of $L_{2}$ into $\partial / \partial x$ and $\partial / \partial y$. However, this fails if $n=3$. Indeed, let

$$
L_{1}=\frac{\partial}{\partial x} \quad \text { and } \quad L_{2}=\frac{\partial}{\partial y}+x \frac{\partial}{\partial z} \quad \text { in } \mathbb{C}^{3}(x, y, z) .
$$

TOME $129-2001-\mathrm{N}^{\mathrm{O}} 4$ 
Then there does not exist $\Phi=\left(\Phi_{1}, \Phi_{2}, \Phi_{3}\right)$ a biholomorphism of $\mathcal{V}_{\mathbb{C}^{3}}(0)$ transforming $L_{1}$ in $\partial / \partial x$ and $L_{2}$ in $\partial / \partial y$, because such $\Phi$ would have to satisfy $\Phi_{3 x}=0$ and $\Phi_{3 y}+x \Phi_{3 z}=0$, whence $\Phi_{3 z}=0, \Phi_{3 y}=0, \Phi_{3}=$ Ct., a contradiction. Furthermore, no multiple of $L_{2}$ can be transformed into $\partial / \partial y$.

The lemma below is analogous to Lemma 8.7 and is also elementary.

LEMMA 8.8. - Let $g \in \mathcal{H}(V), \partial_{z}^{\beta} g \in \mathcal{A}_{\mathcal{F}}(V)$, for all $\beta \in \mathbb{N}^{n}$. Let $q \in H^{1+}$, let $\Lambda:=\Lambda_{\mathcal{F}}(q)$, let $\Lambda^{1+}=\Lambda_{\mathcal{F}^{1+}}(q)(\supset \Lambda)$. Let $z^{+}$be $\mathcal{A}$-coordinates on $\Lambda^{1+}$ and put $g^{+}=g_{\mid \Lambda^{1+}}$. Then $\partial_{z^{+}}^{\beta}\left(g^{+}{ }_{\mid \Lambda}\right)$ is algebraic on $\Lambda$, for all $\beta \in \mathbb{N}^{m+1}$.

Our second main lemma will be as follows. To understand it concretely, the reader may read parallely its formulation in coordinates given in Lemma 8.12 below. With our notations, in an arbitrary fixed leaf $\Lambda_{\mathcal{F}^{1+}}(q)$, this lemma will state that the restriction of $g$ to $\Lambda_{\mathcal{F}^{1+}}(q)$ is algebraic provided it is algebraic on every 'vertical 1-dimensional leaf' $\Lambda_{\mathcal{F}_{L}}(r) \subset \Lambda_{\mathcal{F}^{1+}}(q), r \in \Lambda_{\mathcal{F}}(q)$ and provided all its jets of any order are algebraic, when restricted to the fixed 'horizontal' $m$-dimensional manifold $\Lambda_{\mathcal{F}}(q) \subset \Lambda_{\mathcal{F}^{1+}}(q)$.

Lemma 8.9. - Let $p, V, \mathcal{F}, \mathcal{F}^{1+}$ and $L$ be as above and let $z_{q}^{+} \in \mathbb{C}^{m+1}$ denote some algebraic local coordinates on $\Lambda_{\mathcal{F}^{1+}}(q)$. Let $g \in \mathcal{H}(V, \mathbb{C})$. Then $g \in \mathcal{A}_{\mathcal{F}^{1+}}(V)$, i.e. its restriction $g_{\Lambda_{\mathcal{F}}^{1+}(q)}$ is algebraic for all $q \in H^{1+}$, provided it satisfies the following two conditions for each $q$ :

1) algebraicity on the flow lines of $L: g \in \mathcal{A}_{\mathcal{F}_{L}}(V)$;

2) algebraicity of all the differentials of $g$ after restriction to the central one-codimensional leaf $\Lambda_{\mathcal{F}}(q):\left.\partial_{z_{q}^{+}}^{\beta}\left(g_{\mid \Lambda_{\mathcal{F}}^{1+}(q)}\right)\right|_{\mid \Lambda_{\mathcal{F}}(q)} \in \mathcal{A}\left(\Lambda_{\mathcal{F}}(q), \mathbb{C}\right)$, for all
$\beta \in \mathbb{N}^{m+1}$.

REMARKS. - 1) Notice that the first main assumption of this lemma is already in the hypotheses of Theorem 5.2, and that the second one follows from Lemma 8.6.

2) In coordinates $z_{q}^{+}=\left(z_{q}, w_{q}\right)$ with $\Lambda_{\mathcal{F}}(q)=\left\{w_{q}=0\right\}$, it suffices in fact to require above that all the $\partial_{w_{q}}^{k} g\left(z_{q}, 0\right)$ are algebraic, since then $\partial_{z_{q}}^{\beta} \partial_{w_{q}}^{k} g\left(z_{q}, 0\right)$ are algebraic, by the stability of algebraicity under differentiation. In other words, it suffices to require only that the transversal jets are algebraic, since the horizontal jets are then automatically algebraic.

In conclusion, the foliation $\mathcal{F}^{1+}$ is the sought foliation satisfying $\left({ }^{*}\right)_{m+1}$. Theorem $8.2^{\prime}$ will then be proved once we have proved Lemmas 8.6 and 8.9.

8.10. Proofs of Lemmas 8.6 and 8.9. - It is now possible to reformulate our two main lemmas. Passing to coordinates, there is given an open set $\mathcal{U}=\mathcal{S} \times \mathcal{T} \subset \mathbb{C}^{a} \times \mathbb{C}^{b}$, with, say $\mathcal{S}=\Delta^{a}, \mathcal{T}=\Delta^{b}$, with $a \in \mathbb{N}_{*}, b \in \mathbb{N}_{*}$, $0 \in \mathcal{S}, 0 \in \mathcal{T},(s, t) \in \mathcal{S} \times \mathcal{T}$, and a holomorphic function $g:(s, t) \mapsto g(s, t)$ 
defined over $\mathcal{S} \times \mathcal{T}$. Here, we shall choose $a:=m, b:=n-m$ for Lemma 8.6 and $a:=m, b:=1$ for Lemma 8.9. Let $c:=a+b$.

Lemma 8.11. - If $[\mathcal{S} \ni s \mapsto g(s, t) \in \mathbb{C}] \in \mathcal{A}(\mathcal{S}, \mathbb{C})$, for all $t \in \mathcal{T}$, then there exists a nonempty open subset $\mathcal{T}_{1} \subset \mathcal{T}$ such that $\left[\mathcal{S} \ni s \mapsto \partial_{s, t}^{\gamma} g(s, t) \in \mathbb{C}\right] \in$ $\mathcal{A}(\mathcal{S}, \mathbb{C})$, for all $t \in \mathcal{T}_{1}$, for all $\gamma \in \mathbb{N}^{c}$.

REMARK. - For Lemma 8.6, the sets $\mathcal{S} \times\{t\}$ play the rôle of the leaves of $\mathcal{F}$.

LEMmA 8.12. - If $\left[\mathcal{S} \ni s \mapsto \partial_{t}^{\beta} g(s, 0) \in \mathbb{C}\right] \in \mathcal{A}(\mathcal{S}, \mathbb{C})$ for all $\beta \in \mathbb{N}^{b}$ and if $[\mathcal{T} \ni t \mapsto g(s, t) \in \mathbb{C}] \in \mathcal{A}(\mathcal{T}, \mathbb{C})$, for all $s \in \mathcal{S}$, then $g(s, t) \in \mathcal{A}(\mathcal{S} \times \mathcal{T}, \mathbb{C})$.

REMARK. - For Lemma 8.9, after fixing $q \in H^{1+}$, the set $\mathcal{S} \times\{0\}$ is $\Lambda_{\mathcal{F}}(q)$, the sets $\{s\} \times \mathcal{T}$ are leaves of $\mathcal{F}_{L}$ contained in $\Lambda_{\mathcal{F}}^{1+}(q)$ and $\mathcal{S} \times \mathcal{T}$ is $\Lambda_{\mathcal{F}}^{1+}(q)$.

Proof of Lemma 8.11. - By assumption, for all $t \in \mathcal{T}$, there exists $N_{t} \in \mathbb{N}$, $N_{t} \geq 1, A_{t} \in \mathbb{N}, A_{t} \geq 1, a_{i \alpha, t} \in \mathbb{C}, 1 \leq i \leq N_{t},|\alpha| \leq A_{t}, \alpha \in \mathbb{N}^{a}$ and irreducible polynomials

$$
\mathbb{C}[X, s] \ni P_{t}(X, s):=\sum_{i=0}^{N_{t}} \sum_{|\alpha| \leq A_{t}} a_{i \alpha, t} X^{i} s^{\alpha}
$$

such that

$$
P_{t}(g(s, t), s) \equiv_{s} 0 .
$$

Here, the notation $\Phi(s, t) \equiv_{s} 0$ means that the formal power series $\Phi(s, t)$ vanishes identically when considered as a series in $s$ only. Let us transform the $a_{i \alpha, t}$ first to make them depend in a nice way with respect to $t$.

LEMMA 8.14. - Then there exists a nonempty open set $\mathcal{T}_{1} \subset \subset \mathcal{T}$ and an irreducible polynomial of uniformly bounded degree having coefficients $a_{i \alpha}(t)$ holomorphic over $\mathcal{T}_{1}$, namely there exists:

$$
\mathcal{H}\left(\mathcal{T}_{1}\right)[X, s] \ni P(X, s ; t):=\sum_{i=0}^{N} \sum_{|\alpha| \leq A} a_{i \alpha}(t) X^{i} s^{\alpha}, \quad a_{i \alpha} \in \mathcal{H}\left(\mathcal{T}_{1}\right),
$$

such that $P(g(s, t), s ; t) \equiv_{s, t} 0$, for all $s \in \mathcal{S}$ and $t \in \mathcal{T}_{1}$.

End of proof of Lemma 8.11. - Taking Lemma 8.14 for granted, it then suffices to differentiate $P(g(s, t), s ; t) \equiv 0$ with respect to $t$, namely to apply $\partial_{t}^{\beta}$, $|\beta|=1$, and to eliminate $g(s, t)$ from the system of algebraic equations:

$$
\left\{\begin{array}{l}
\sum_{i=0}^{N} \sum_{|\alpha| \leq A}\left(\partial_{t}^{\beta} a_{i \alpha}(t) g(s, t)+i a_{i \alpha}(t) \partial_{t}^{\beta} g(s, t)\right) g(s, t)^{i-1} s^{\alpha}=0, \\
P(g(s, t), s ; t)=0
\end{array}\right.
$$

this giving the algebraicity of $s \mapsto \partial_{t}^{\beta} g(s, t)$ for all $|\beta|=1$. General induction is analogous: simply replace $g(s, t)$ by $\partial_{t}^{\beta} g(s, t)$.

TOME $129-2001-\mathrm{N}^{\mathrm{O}} 4$ 
Proof of Lemma 8.14. - To prove the assertion, consider the countable many sets

$$
\mathcal{E}_{N, A}=\left\{t \in \mathcal{T} ; N_{t}=N, A_{t}=A\right\}
$$

whose union $\bigcup_{N \geq 1, A \geq 1} \mathcal{E}_{N, A}$ equals $\mathcal{T}$, by (8.13). Of course, the union of their closures

$$
\bigcup_{N \geq 1, A \geq 1} \overline{\mathcal{E}_{N, A}}
$$

equals $\mathcal{T}$ as well. Thanks to Baire's category theorem, at least one closure $\overline{\mathcal{E}_{N, A}}$ has nonempty interior. This is the main trick. Thus, there exists a nonempty open polydisc $\mathcal{T}_{1} \subset \subset \mathcal{T}$ with center $t_{1} \in \mathcal{T}_{1}$ such that $\mathcal{T}_{1} \subset \overline{\mathcal{E}_{N, A}}$. Then we have polynomial relations:

$$
P_{t}(g(s, t), s)=\sum_{i=0}^{N} \sum_{|\alpha| \leq A} a_{i \alpha, t} g(s, t)^{i} s^{\alpha} \equiv_{s} 0, \quad \text { for } t \in \mathcal{T}_{1} \cap \mathcal{E}_{N, A},
$$

with a uniform bound for the degrees on a dense subset of $\mathcal{T}_{1}$. This means that the finite set of functions

$$
\mathcal{C}_{t}=\left\{\mathcal{S} \ni s \mapsto g(s, t)^{i} s^{\alpha} \in \mathbb{C}\right\}_{0 \leq i \leq N,|\alpha| \leq A}
$$

is always linearly dependent for $t \in \mathcal{T}_{1} \cap \mathcal{E}_{N, A}$, and in particular for $t=t_{1}$. Let us normalize one $a_{i_{*} \alpha_{*}, t_{1}}=1$, for some $i_{*}, \alpha_{*}$ and let us denote, after renumbering its elements, the set $\mathcal{C}_{t}$ by $\mathcal{C}_{t}:=\left\{\mathcal{S} \ni s \mapsto h_{k}(s, t) \in \mathbb{C}\right\}_{1 \leq k \leq B}$, with $B \in \mathbb{N}_{*}$ and

$$
h_{B}(s, t)=: g^{i_{*}}(s, t) s^{\alpha_{*}} .
$$

Further, let us develope each $h_{k}$ in power series with respect to $s$ :

$$
h_{k}(s, t)=\sum_{\ell \in \mathbb{N}^{a}} h_{k \ell}(t) s^{\ell}, \quad h_{k \ell} \in \mathcal{H}\left(\mathcal{T}_{1}\right) .
$$

LEMma 8.20. - Now, the following properties hold:

1) The functions $h_{1}\left(s, t_{1}\right), \ldots, h_{B-1}\left(s, t_{1}\right)$ are linearly independent over $\mathbb{C}$.

2) There exist $B-1$ pairwise distinct multiindices $\ell_{* 1}, \ldots, \ell_{* B-1} \in \mathbb{N}^{a}$ such that the determinant $\operatorname{det}\left(\left(h_{k \ell_{* j}}\left(t_{1}\right)\right)_{1 \leq k, j \leq B-1}\right)$ is nonzero.

3) Consequently, the functions of $s, h_{1}(s, t), \ldots, h_{B-1}(s, t)$ are linearly independent over $\mathbb{C}$ for all $t$ running in a neighborhood of $t_{1}$.

Proof. - Suppose by contradiction that there exist $d_{1}, \ldots, d_{B-1} \in \mathbb{C}$ not all zero such that $d_{1} h_{1}\left(s, t_{1}\right)+\cdots+d_{B-1} h_{B-1}\left(s, t_{1}\right) \equiv_{s} 0$. Reexpressing the $h_{k}$ 's in terms of $g$ and $s$, this means that there exists a nonzero polynomial $Q(X, s)$ such that

$$
Q\left(g\left(s, t_{1}\right), s\right) \equiv{ }_{s} 0 .
$$

BULLETIN DE LA SOCIÉtÉ MATHÉMATIQUE DE FRANCE 
As $P_{t_{1}}(X, s)$ was assumed to be irreducible, it follows that there exists a nonzero polynomial $u(X, s)$ such that

$$
Q(X, s) \equiv u(X, s) P_{t_{1}}(X, s) .
$$

But the monomials of $Q$ are exactly the same as those of $P_{t_{1}}$, except one missing term $X^{i_{*}} s^{\alpha_{*}}$. For reasons of degree, $u$ is then a nonzero constant. This contradicts the fact that $P_{t_{1}}$ incorporates the monomial $X^{i_{*}} s^{\alpha_{*}}$, since $a_{i_{*}, \alpha_{*}, t_{1}}=1$ by our previous choice. Thus, 1$)$ is proved. Then the $(B-1) \times \infty$-matrix of complex coefficients $\left(h_{k \ell}\left(t_{1}\right)\right)_{1 \leq k \leq B-1, \ell \in \mathbb{N}^{a}}$ possesses a nonzero $(B-1) \times(B-1)$ minor, which yields 2 ) and then 3 ) evidently.

End of proof of Lemma 8.14. - Shrinking $\mathcal{T}_{1}$ if necessary, we can therefore assume that the functions $h_{1}(s, t), \ldots, h_{B-1}(s, t)$ are linearly independent for all $t \in \mathcal{T}_{1}$. Remembering that $h_{1}(s, t), \ldots, h_{B}(s, t)$ are, on the contrary, linearly dependent for all $t \in \mathcal{T}_{1} \cap \mathcal{E}_{N, A}$, we obtain that there exist $a_{1, t}, \ldots, a_{B-1, t} \in \mathbb{C}$ such that

$$
a_{1, t} h_{1}(s, t)+\cdots+a_{B-1, t} h_{B-1}(s, t) \equiv_{s} h_{B}(s, t), \quad \forall t \in \mathcal{T}_{1} \cap \mathcal{E}_{N, A},
$$

or equivalently, using the developement (8.19),

$$
a_{1, t} h_{1 l}(t)+\cdots+a_{B-1, t} h_{B-1 \ell}(t)=h_{B \ell}(t), \quad \forall t \in \mathcal{T}_{1} \cap \mathcal{E}_{N, A}, \forall \ell \in \mathbb{N}^{a}
$$

Writing in particular these equations for $\ell=\ell_{1 *}, \ldots, \ell_{B-1 *}$, using property 2) of Lemma 8.20 and Cramer's rule, we deduce that the coefficients $a_{1, t}, \ldots, a_{B-1, t}$ can be uniquely expressed as rational functions with respect to the $h_{k, \ell_{* j}}(t), k=1, \ldots, B, j=1, \ldots, B-1$, with the denominator $\operatorname{det}\left(\left(h_{k \ell_{* j}}(t)\right)_{1 \leq k, j \leq B-1}\right)$, nonvanishing over $\mathcal{T}_{1}$. We thus have got holomorphic functions $a_{1}(t), \ldots, a_{B-1}(t)$ over $\mathcal{T}_{1}$ which are rational in the coefficients $h_{k, \ell_{* j}}(t)$ and which satisfy

$$
a_{1}(t) h_{1}(s, t)+\cdots+a_{B-1}(t) h_{B-1}(s, t) \equiv{ }_{s} h_{B}(s, t),
$$

for all $t \in \mathcal{T}_{1} \cap \mathcal{E}_{N, A}$ and then for all $t \in \mathcal{T}_{1}$, by continuity. Reexpressing the $h_{k}$ 's in terms of $g$ and $s$, we finally get (8.15).

Proof of Lemma 8.12. - Direct differentiations with respect to $t$ yield the following more explicit version of (8.19), where the rôles of $s$ and $t$ are exchanged:

LEMMA 8.24. - There exist integers $c_{k, \beta, \gamma}$ and universal polynomials $\phi_{k, \beta, \gamma}$ such that each monomial $g(s, t)^{k} t^{\beta}$ can be expressed as

$$
g(s, t)^{k} t^{\beta}=\sum_{\gamma \in \mathbb{N}^{b}} \phi_{k, \beta, \gamma}\left(\left\{\partial_{t}^{\beta} g(s, 0)\right\}_{|\beta| \leq c_{k, \beta, \gamma}}\right) t^{\gamma} .
$$

TOME $129-2001-\mathrm{N}^{\mathrm{O}} 4$ 
Proof. - Applying the differentiations $\partial_{t}^{\gamma} \mid t=0$ to $g(s, t)^{k} t^{\beta}$, we simply get

$$
\phi_{k, \beta, \gamma}\left(\left\{\partial_{t}^{\beta} g(s, 0)\right\}_{|\beta| \leq c_{k, \beta, \gamma}}\right):=\left.\partial_{t}^{\gamma}\right|_{t=0}\left(g(s, t)^{k} t^{\beta}\right) / \gamma ! .
$$

This expression is very appropriate, because the partial derivatives $\partial_{t}^{\beta} g(s, 0)$ appear in an algebraic way. On the other hand, the maps $t \mapsto g(s, t)$ are algebraic by assumption. Inversing the rôles of $s$ and $t$, Lemma 8.14 then yields an open subset $\mathcal{S}_{1} \subset \subset \mathcal{S}$ and an irreducible polynomial

$$
R(Y, t ; s)=\sum_{k=0}^{N} \sum_{\beta \in \mathbb{N}^{b},|\beta| \leq B} b_{k \beta}(s) Y^{k} t^{\beta}
$$

such that $R(g(s, t), t ; s) \equiv_{t, s} 0$. Here, the functions $b_{k \beta}(s)$, which are holomorphic over $\mathcal{S}_{1}$, are in fact rational with respect to a finite number of the coefficients $\phi_{\ell, \beta, \gamma}\left(\left\{\partial_{t}^{\beta} g(s, 0)\right\}_{|\beta| \leq c_{\ell, \beta, \gamma}}\right)$ appearing in (8.25), as we have observed just after (8.22) in our application of Cramer's rule. In summary, there exist an integer $e \in \mathbb{N}_{*}$ and a polynomial relation

$$
R(g(s, t), t)=\sum_{\beta \in \mathbb{N}^{b},|\beta| \leq B} \sum_{k=0}^{N} \psi_{k \beta}\left(\left\{\partial_{t}^{\beta} g(s, 0)\right\}_{|\beta| \leq e}\right) g(s, t)^{k} t^{\beta} \equiv_{s, t} 0,
$$

with the $\psi_{k \beta}$ being rational. As by assumption, the partial derivatives $\partial_{t}^{\beta} g(s, 0)$ are all algebraic, using elimination theory, we can transform (8.27) into a polynomial relation $S(g(s, t), s, t)=0$, where $S(Z, s, t) \in \mathbb{C}[Z, s, t]$, which shows that $g(s, t)$ is algebraic. The proofs of Lemmas 8.14 and 8.12 are complete.

In conclusion, Theorem 5.2 and Theorem 2.5 are now fully established. It remains now to explain what is the degree of algebraic degeneracy.

\section{Algebraic degeneracy}

9.1. Real algebraic sets. - We denote by $\mathbb{C}[z, \bar{z}]_{\mathbb{R}} \subset \mathbb{C}[z, \bar{z}]$ the ideal of real polynomials $P(z, \bar{z})$, namely those satisfying $P(z, \bar{z}) \equiv \bar{P}(\bar{z}, z)$. Then $\mathbb{C}[z, \bar{z}]_{\mathbb{R}}$ is isomorphic to $\mathbb{R}[\operatorname{Re} z, \operatorname{Im} z]$. Let $\Sigma \subset \mathbb{C}^{n}$ be a real algebraic set, defined as the zero set of a collection of elements of $\mathbb{C}[z, \bar{z}]_{\mathbb{R}}$. Let $\mathcal{J}(\Sigma)$ denote the ideal of polynomials vanishing on $\Sigma$. This ideal is prime if and only if $\Sigma$ is irreducible, which we will suppose throughout $\S 9$. The field of fraction $K(\Sigma)$ of the entire ring $\mathbb{C}[z, \bar{z}]_{\mathbb{R}} / \mathcal{J}(\Sigma)$ is called the field of rational functions on $\Sigma$. Its transcendence degree over $\mathbb{C}$ is called the dimension of $\Sigma$. Let $\delta$ be this dimension. If $P_{1}, \ldots, P_{\sigma} \in \mathbb{C}[z, \bar{z}]_{\mathbb{R}}$ is a system of generators of the prime ideal $\mathcal{J}(\Sigma)$, the generic rank of the complex $\sigma \times 2 n$ Jacobian matrix

$$
J_{P}(z, \bar{z}):=\left(\frac{\partial P_{i}}{\partial z_{k}}(z, \bar{z}) \frac{\partial P_{i}}{\partial \bar{z}_{k}}(z, \bar{z})\right)_{1 \leq i \leq \sigma, 1 \leq k \leq n},
$$

BULLETIN DE LA SOCiÉtÉ MATHÉMATIQUE DE FRANCE 
is then equal to $d:=n-\delta$ over $\Sigma$, the codimension of $\Sigma$. The set $\Sigma_{\text {reg }}$ at which the rank of $J_{P}(z, \bar{z})$ is equal to $d$ does not depend on the choice of a system of generators for $\mathcal{J}(\Sigma)$ and is called the set of regular points of $\Sigma$, in the algebraic sense. Its complement

$$
\Sigma_{\text {sing }}:=\Sigma \backslash \Sigma_{\text {reg }}
$$

is a proper real algebraic subvariety of $\Sigma$ called its set of singular points.

EXAMPLE 9.3. - The celebrated Whitney umbrella is the cubic of $\mathbb{R}^{3}$ defined by

$$
W:=\left\{\left(x_{1}, x_{2}, x_{3}\right) \in \mathbb{R}^{3}: x_{3} x_{1}^{2}=x_{2}^{2}\right\} .
$$

In the algebraic sense, $W_{\text {sing }}=0 \times 0 \times \mathbb{R}$ and the set

$$
W_{\text {reg }}=W \cap\left\{\left(x_{1}, x_{2}\right) \neq(0,0)\right\} \subset\left\{x_{1} \neq 0\right\}
$$

is not connected and not dense in $W$ for the euclidean topology. This example also shows that the set of geometrically singular points of $W$, equal to $\left\{\left(0,0, x_{3}\right): x_{3} \geq 0\right\}$ is only semi-algebraic. Let us consider the tube $\Sigma:=W \times(i \mathbb{R})^{3}$ over $W$ in $\mathbb{C}^{3}$, namely

$$
\Sigma:=\left\{\left(z_{1}, z_{2}, z_{3}\right) \in \mathbb{C}^{3}: x_{3} x_{1}^{2}=x_{2}^{2}\right\}, \quad x_{j}=\operatorname{Re} z_{j} .
$$

The set $\Sigma_{\text {sing }}=\left\{x_{1}=x_{2}=0\right\}=\mathbb{R} \times \mathbb{R} \times \mathbb{C}$ is then 1-algebraically degenerate and nowhere minimal. The set $\Sigma_{\text {reg }}=\Sigma \cap\left\{x_{1} \neq 0\right\}$ is a rigid real algebraic hypersurface globally defined as the graph $x_{3}=x_{2}^{2} / x_{1}^{2}$ on $\mathbb{C}^{3} \backslash\left\{x_{1}=0\right\}$ which carries the two $\mathrm{CR}$ vector fields

$$
\bar{L}_{1}:=x_{1} \frac{\partial}{\partial \bar{z}_{1}}-2 x_{3} \frac{\partial}{\partial \bar{z}_{3}} \quad \text { and } \quad \bar{L}_{2}:=x_{1}^{2} \frac{\partial}{\partial \bar{z}_{2}}+2 x_{2} \frac{\partial}{\partial \bar{z}_{3}} .
$$

Since the Lie bracket

$$
\left[L_{2}, \bar{L}_{2}\right]=x_{1}^{2} \frac{\partial}{\partial \bar{z}_{3}}-x_{1}^{2} \frac{\partial}{\partial z_{3}}
$$

is linearly independent with $\left(\bar{L}_{1}, \bar{L}_{2}\right)$ at every point, the hypersurface $\Sigma_{\text {reg }}$ is minimal at every point. Further, if

$$
\mathcal{T}:=\sum_{j=1}^{3} a_{j}(z) \frac{\partial}{\partial z_{j}}
$$

is a local holomorphic algebraic vector field tangent to $\Sigma_{\text {reg }}$, we have

$$
a_{1}(z) x_{1} x_{3}-a_{2}(z) x_{2}+\frac{1}{2} a_{3}(z) x_{1}^{2}=0,
$$

whence $a_{1}=a_{2}=a_{3}=0$, namely $\Sigma_{\text {reg }}$ is algebraically nondegenerate (it is even Levi-nondegenerate at every point). This contrasts with $\Sigma_{\text {sing }}$.

Let us finally illustrate Theorems $2.5,2.6$ and 3.1. Let $f:(\Sigma, p) \rightarrow(\Sigma, p)$ be a germ of biholomorphism fixing $p$. If $p \in \Sigma_{\text {sing }} \cap\left\{x_{3}<0\right\}$, there exists a local perturbation $\phi \circ f$ of $f$ such that $\nabla^{\operatorname{tr}}(\phi \circ f)=1$. If $p \in \Sigma \cap\left\{x_{3} \geq 0\right\}$, then $f$ is necessarily algebraic. 
9.4. Extrinsic complexification. - We define

$$
\Sigma^{c}:=\left\{(z, \zeta) \in \mathbb{C}^{2 n}: P(z, \zeta)=0, \forall P \in \mathcal{J}(\Sigma)\right\}
$$

Then $\Sigma^{c}$ is a complex algebraic subset of $\mathbb{C}^{2 n}$ with $\Sigma^{c} \cap \underline{\Lambda}=\Sigma$, where

$$
\underline{\Lambda}=\{\zeta=\bar{z}\}
$$

if we identify $\{(z, \bar{z}): z \in \Sigma\}$ with $\{z \in \Sigma\}$. This set $\Sigma^{c}$ is useful because $\Sigma_{\text {reg }}$ need not be connected. Using the rank property of the Jacobian (9.2) and the connectedness of the regular part of complex algebraic sets, one can check that $\Sigma^{c}$ is irreducible if $\Sigma$ is. Further, using the connectedness of the regular part $\Sigma_{\text {reg }}^{c}$ of $\Sigma^{c}$, one can show that if there exists a point $p \in \Sigma$ such that $r(p, \bar{p}) \neq 0$, where $r(z, \bar{z}) \in \mathbb{C}[z, \bar{z}]_{\mathbb{R}}$, then the set $\{z \in \Sigma: r(z, \bar{z}) \neq 0\}$ is dense in $\Sigma_{\text {reg }}$ for the euclidean topology. An arbitrary union of sets of the form $\{z \in \Sigma: r(z, \bar{z}) \neq 0\}$, where $r$ does not vanish identically on $\Sigma$, will be called a Zariski open subset of $\Sigma$.

A point $p \in \Sigma$ will be called Zariski-generic if it runs in some Zariski open subset of $\Sigma$.

9.5. Intrinsic complexification. - By noetherianity of $\mathbb{C}[z]$, the intersection of all complex algebraic subvarieties containing $\Sigma$ is a complex algebraic subvariety, called the intrinsic complexification of $\Sigma$ and denoted by $\Sigma^{i_{c}}$. Again, passing to extrinsic complexification, from the relation $\left(\Sigma^{i_{c}}\right)^{c}=\Sigma^{i_{c}} \times \mathbb{C}_{\zeta}^{n}$ and using the principle of analytic continuation for complex algebraic subsets, it follows that $\Sigma^{i_{c}}$ is irreducible.

9.6. Locus of CR points. - We denote by $\Sigma_{\mathrm{CR}}$ the set of points $z \in \Sigma_{\text {reg }}$ at which the rank of the $\sigma \times n$ complex matrix $\left(\partial P_{i} / \partial z_{k}(z, \bar{z})\right)_{1 \leq i \leq \sigma, 1 \leq k \leq n}$ is maximal, hence locally constant. It is called the set of $C R$ points of $\Sigma$.

Of course, its extrinsic complexification $\left(\Sigma_{\mathrm{CR}}\right)^{c}$, the set of $(z, \zeta) \in \Sigma_{\text {reg }}^{c}$ at which the complex matrix $\left(\partial P_{i} / \partial z_{k}(z, \zeta)\right)_{1 \leq k \leq n, 1 \leq i \leq \sigma}$ is maximal and locally constant, is also connected. Let $d_{1}$ be this generic point. We have $0 \leq d_{1} \leq d$. Notice that $\Sigma$ is CR-generic at a Zariski-generic rank if and only if $d_{1}=d$. Let the CR-codimension $\operatorname{codim}_{\mathrm{CR}} \Sigma$ be $d-d_{1}$. It is well known that in a neighborhood of a point $p \in \Sigma_{\mathrm{CR}}$, then $\Sigma$ is contained in a holomorphic algebraic submanifold of codimension $\left(d-d_{1}\right)$, which is the local intrinsic complexification $\Sigma_{\mathrm{loc}}^{\mathrm{ic}}$ of $\Sigma$. Clearly, $\Sigma_{\mathrm{loc}}^{\mathrm{ic}}$ is contained in a unique irreducible complex algebraic subset of $\mathbb{C}^{n}$. Passing to extrinsic complexification to use the connectedness of $\Sigma^{c}$, one can check that this irreducible complex algebraic subset does not depend on $p \in \Sigma_{\mathrm{CR}}$ and is nothing else than the (global) intrinsic complexification $\Sigma^{i_{c}}$ defined in $\S 9.5$ above. 
9.7. Nonlocality of algebraic degeneracy. - At an arbitrary point $p \in \Sigma_{\mathrm{CR}}$, we define the integer $\kappa_{\Sigma, p}$ to be the maximal number of $(1,0)$ vector fields $L_{1}, \ldots, L_{j}$ with algebraic coefficients in a neighborhood of $p$ which induce a complex algebraic foliation of $\mathcal{V}_{\mathbb{C}^{n}}(p)$ (possibly with singularities; of course, algebraic foliation implies algebraicity of the coefficients) such that

1) $L_{1}, \ldots, L_{j}$ are tangent to $\Sigma_{\mathrm{CR}} \cap \mathcal{V}_{\mathbb{C}^{n}}(p)$;

2) $L_{1}, \ldots, L_{j}$ are linearly independent: if $b_{1}, \ldots, b_{j}$ are algebraic holomorphic in $\mathcal{V}_{\mathbb{C}^{n}}(p)$ and if $b_{1} L_{1}+\cdots+b_{j} L_{j}=0$, then $b_{1}=\cdots=b_{j}=0$.

Then the vectors $L_{1}(q), \ldots, L_{\kappa_{\Sigma, p}}(q)$ are $\mathbb{C}$-linearly independent for $q$ varying in a Zariski open subset of $\Sigma_{\mathrm{CR}} \cap \mathcal{V}_{\mathbb{C}^{n}}(p)$. Evidently, the function $\Sigma_{\mathrm{CR}} \ni p \mapsto$ $\kappa_{\Sigma, p} \in \mathbb{N}$ is lower semi-continuous.

Lemma 9.8. - Let $p, q \in \Sigma_{\mathrm{CR}}$. Then $\kappa_{\Sigma, p}=\kappa_{\Sigma, q}$.

Proof. - Let $L_{1}, \ldots, L_{\kappa \Sigma, p}$ satisfy 1) and 2) above in $\mathcal{V}_{\mathbb{C}^{n}}(p)$. The coefficients of

$$
L_{j}:=\sum_{k=1}^{n} a_{j, k}(z) \frac{\partial}{\partial z_{k}}
$$

being holomorphic algebraic in a neighborhood of $p, j=1, \ldots, \kappa_{\Sigma, p}$, there exists a complex algebraic subvariety $E$ of $\mathbb{C}^{n}$ with $p \notin E$, such that

3) the coefficients $a_{j, k}(z)$ extend holomorphically along any continuous path $\gamma$ with origin $p$ which is contained in $\mathbb{C}^{n} \backslash E$ and with endpoint an arbitrary point $q \in \mathbb{C}^{n} \backslash E$;

4) the extended vector fields at $q$ have holomorphic algebraic coefficients, they induce an algebraic foliation and they are linearly independent at $q$ in the sense of 2) above.

Notice that the vector fields $\left(L_{1}\right)^{c}, \ldots,\left(L_{\kappa_{\Sigma, p}}\right)^{c}$ are tangent to $\left(\Sigma_{\mathrm{CR}}\right)^{c}$ in a neighborhood of $p$. Let $q \in \Sigma_{\mathrm{CR}}$ be arbitrary. Remember that if $A, B$ are complex algebraic sets with $A$ irreducible, $A_{\text {reg }} \backslash B$ is connected and nonempty unless $B$ contains $A$. Hence $\left(\Sigma_{\mathrm{CR}}\right)^{c} \backslash\left(E \times \mathbb{C}_{\zeta}^{n}\right)$ is connected. By 3), there exists a continous path $\gamma$ from $(p, \bar{p})$ to $(q, \bar{q})$ running in $\left(\Sigma_{\mathrm{CR}}\right)^{c} \backslash\left(E \times \mathbb{C}_{\zeta}^{n}\right)$. By the principle of analytic continuation, it follows that the extended vector fields along $\gamma$, say $L_{1}^{\prime}, \ldots, L_{\kappa_{\Sigma, p}}^{\prime}$ are tangent to $\left(\Sigma_{\mathrm{CR}}\right)^{c} \cap \mathcal{V}_{\mathbb{C}^{2 n}}(q, \bar{q})$. We deduce $\kappa_{\Sigma, p} \leq \kappa_{\Sigma, q}$, and then $\kappa_{\Sigma, p}=\kappa_{\Sigma, q}$, as desired.

9.9. Local straightening property. - Let $\kappa_{\Sigma}$ denote the common value of all the $\kappa_{\Sigma, p}$ for $p \in \Sigma_{\mathrm{CR}}$ and call it the degree of algebraic degeneracy of $\Sigma$. The following statement achieves to explain Theorem 2.5.

THEOREM 9.10. - There exists a proper real algebraic subvariety $F$ of $\Sigma$ with $\Sigma \backslash F \subset \Sigma_{\mathrm{CR}}$ such that in a neighborhood of every point $p \in \Sigma \backslash F$, there exist local holomorphic algebraic coordinates in which $\Sigma$ is of the form $\Delta^{\kappa_{\Sigma}} \times \Sigma_{p}$, where $\Sigma_{p} \subset \mathbb{C}^{n-\kappa_{\Sigma}}$ is a piece of smooth CR real algebraic subset satisfying $\kappa_{\Sigma_{p}}=0$.

TOME $129-2001-\mathrm{N}^{\mathrm{O}} 4$ 
Proof. - We check first $\kappa_{\underline{\Xi}_{p}}=0$. Otherwise, let $\underline{L}$ be a $(1,0)$ vector field in $\mathbb{C}^{n-\kappa_{\Sigma}}$ inducing an algebraic foliation tangent to $0 \times \underline{\Sigma}_{p}$. Of course, $\underline{L}$ is linearly independent with $L_{1}=\partial / \partial z_{1}, \ldots, L_{\kappa_{\Sigma}}=\partial / \partial z_{\kappa_{\Sigma}}$. This contradicts $\kappa_{\Sigma, p}=\kappa_{\Sigma}$.

Let us now establish the product property. According to the preceding considerations, there exist multivalued global vector fields $L_{1}, \ldots, L_{\kappa_{\Sigma}}$ defined outside a complex algebraic subset $E$ of $\mathbb{C}^{n}$. Enlarging $E$ if necessary, we can assume that the vectors $L_{1}(q), \ldots, L_{\kappa_{\Sigma}}(q)$ are linearly independent at every point $q \in \mathbb{C}^{n} \backslash E$. Let $\Sigma_{N C R} \supset \Sigma_{\text {sing }}$ be the set of non-CR points of $\Sigma$. Clearly, $\Sigma_{N C R}$ is a proper real algebraic subvariety of $\Sigma$. We set

$$
F:=\Sigma_{N C R} \cup(E \cap \Sigma) .
$$

According to $\S 9.6$ above, $\Sigma_{\mathrm{CR}}$ is CR-generic in its local intrinsic complexification $\left(\Sigma_{\mathrm{CR}} \cap \mathcal{V}_{\mathbb{C}^{n}}(p)\right)^{i_{c}}=\Sigma^{i_{c}} \cap \mathcal{V}_{\mathbb{C}^{n}}(p)$, which is a smooth complex algebraic subvariety of codimension equal to $\operatorname{codim}_{\mathrm{CR}} \Sigma^{i_{c}}$. The vector fields $L_{1}, \ldots, L_{\kappa_{\Sigma}}$ being tangent to $\Sigma_{\mathrm{CR}}$, their restriction to $\Sigma^{i_{c}} \cap \mathcal{V}_{\mathbb{C}^{n}}(p)$ is tangent to it. To prove Theorem 9.10, we can therefore reason directly inside $\Sigma^{i_{c}} \cap \mathcal{V}_{\mathbb{C}^{n}}(p)$. In other words, we come to the following statement.

Lemma 9.11. - Let $M \subset \mathbb{C}^{n}$ be a $\mathrm{CR}$-generic real algebraic submanifold, let $p \in M$ and let $L_{1}, \ldots, L_{\kappa}, \kappa \geq 1$, be $(1,0)$ vector fields with algebraic coefficients such that

1) the vectors $L_{1}(q), \ldots, L_{\kappa}(q)$ are linearly independent;

2) the induced foliations $\mathcal{F}_{L_{1}}, \ldots, \mathcal{F}_{L_{\kappa}}$ are algebraic in $\mathcal{V}_{\mathbb{C}^{n}}(p)$;

3) the vector fields $L_{1}, \ldots, L_{\kappa}$ are tangent to $M \cap \mathcal{V}_{\mathbb{C}^{n}}(p)$.

Then there exist local algebraic coordinates at $p$ in which $M=\Delta^{\kappa} \times \underline{M}$, where $\underline{M} \subset \mathbb{C}^{n-\kappa}$ is a CR-generic real algebraic submanifold.

Proof. - According to $\S 6.1$, there exist local coordinates $t=(w, z) \in \mathbb{C}^{m} \times \mathbb{C}^{d}$ in which $p=0$ and $M: z_{\ell}=\bar{Q}_{\ell}(w, \bar{t}), \ell=1, \ldots, d$. We shall identify $\left(t_{1}, \ldots, t_{m}\right)=\left(w_{1}, \ldots, w_{m}\right)$. Of course $\kappa \leq m$. Let

$$
L_{1}=\sum_{k=1}^{n} a_{k}(t) \frac{\partial}{\partial t_{k}}
$$

We can assume that $a_{1}(0) \neq 0$. Since $a_{k} / a_{1}$ is still holomorphic algebraic, $k=2, \ldots, n$, we come down to

$$
L_{1}=\frac{\partial}{\partial w_{1}}+\sum_{k=2}^{n} a_{k}(t) \frac{\partial}{\partial t_{k}}
$$

Due to $a_{1}(t) \equiv 1$, the following lemma puts in concrete form the assumption of algebraicity of $\mathcal{F}_{L_{1}}$.

Lemma 9.12. - The foliation $\mathcal{F}_{L_{1}}$ is algebraic if and only if the flow of $\mathcal{F}_{L_{1}}$ is algebraic.

BULletin DE LA SOCiÉtÉ MATHÉMATIQUE DE FRANCE 
Proof. - For small $u \in \mathbb{C}$ and $t \in \mathbb{C}^{n}$, let $\varphi_{1}(u, t):=\exp \left(u L_{1}\right)(t)$, be the local flow of $L_{1}$ in a neighborhood of 0 . If it is algebraic, then the algebraic biholomorphism $\Phi\left(w_{1}, t_{2}, \ldots, t_{n}\right):=\varphi_{1}\left(w_{1}, 0, t_{2}, \ldots, t_{n}\right)$ produces some new straightened algebraic coordinates in which the integral curves of $L_{1}$ are the affine lines $\left\{t_{2}=c_{2}, \ldots, t_{n}=c_{n}\right\}$, since $\Phi_{*}\left(\partial / \partial w_{1}\right)=L_{1}$. This shows the algebraicity of $\mathcal{F}_{L_{1}}$.

Conversely, let $\Phi: \mathbb{C} \times \mathbb{C}^{n-1} \ni(x, y) \mapsto \Phi(x, y) \in \mathbb{C}^{n}, \Phi(0)=0$, be a local algebraic coordinate system in which the lines $\{y=c\}$ coincide with the complex integral curves of $L_{1}$. As $a_{1}(t) \equiv 1$, for fixed $u_{*}$, the flow of $L_{1}$ at time $u_{*}$, restricted to the algebraic hypersurface $\left\{w_{1}=0\right\}$, namely the map $\left(t_{2}, \ldots, t_{n}\right) \mapsto \varphi_{1}\left(u_{*}, 0, t_{2}, \ldots, t_{n}\right)$, sends $\left\{w_{1}=0\right\}$ onto the algebraic hypersurface $\left\{w_{1}=u_{*}\right\}$. Thanks to the coordinates defined by $\Phi$, we deduce that

1) the maps $\left(t_{2}, \ldots, t_{n}\right) \mapsto \varphi_{1}\left(w_{1 *}, 0, t_{2}, \ldots, t_{n}\right)$ are algebraic;

2) the maps $w_{1} \mapsto \varphi_{1}\left(w_{1}, 0, t_{2 *}, \ldots, t_{n *}\right)$ are algebraic.

According to the separate algebraicity principle [3], the map $\left(w_{1}, t_{2}, \ldots, t_{n}\right) \mapsto$ $\varphi_{1}\left(w_{1}, 0, t_{2}, \ldots, t_{n}\right)$ is then algebraic. Thanks to $a_{1}(t) \equiv 1$, we have

$$
\varphi_{1}\left(w_{1}, t_{1}, t_{2}, \ldots t_{n}\right) \equiv \varphi_{1}\left(w_{1}+t_{1}, 0, t_{2}, \ldots, t_{n}\right),
$$

so the total flow of $L_{1}$ is algebraic, as desired.

End of proof of Lemma 9.11. - Since the straightened field $\partial / \partial w_{1}$ is tangent to $M$, in coordinates like in $\S 6.1$, the functions $\bar{Q}_{\ell}$ are independent of $\left(w_{1}, \bar{w}_{1}\right)$. Thus $M$ is the product $\Delta \times \underline{M}$, where $\underline{M} \subset \mathbb{C}^{n-1}$ is given by

$$
z_{\ell}=\bar{Q}_{\ell}\left(w_{2}, \ldots, w_{m}, \bar{t}_{2}, \ldots, \bar{t}_{n}\right), \quad \ell=1, \ldots, d .
$$

If $\kappa \geq 2$, let

$$
L_{2}=\sum_{k=1}^{n} a_{k}(t) \frac{\partial}{\partial t_{k}}
$$

be linearly independent with $\partial / \partial w_{1}$ at 0 . We can assume that $a_{2}(t) \equiv 1$, namely

$$
L_{2}=a_{1}(t) \frac{\partial}{\partial w_{1}}+\frac{\partial}{\partial w_{2}}+\sum_{k=3}^{n} a_{k}(t) \frac{\partial}{\partial t_{k}} .
$$

Thanks to Lemma 9.12 above, its flow $\varphi_{2}(u, t):=\exp \left(u L_{2}\right)(t)$ is algebraic. Then

$$
\Phi\left(t_{2}, t_{3}, \ldots, t_{n}\right):=\exp \left(t_{2} L_{2}\right)\left(0,0, t_{3}, \ldots, t_{n}\right)
$$

is algebraic. Denote

$$
\Phi:=\left(\Phi_{1}, \Phi_{2}, \ldots \Phi_{n}\right) \text { and } \underline{\Phi}:=\left(\Phi_{2}, \ldots, \Phi_{n}\right) .
$$

We have $\operatorname{det}\left(\partial \underline{\Phi}_{j} / \partial t_{k}(0)\right)_{2 \leq j, k \leq n} \neq 0$. The map $\underline{\Phi}$ therefore induces a local algebraic biholomorphism of $0 \times \mathbb{C}^{n-1}$ fixing 0 . Since $L_{2}$ is tangent to $M$, we have

TOME $129-2001-\mathrm{N}^{\mathrm{O}} 4$ 
$\left(0,0, t_{3}, \ldots, t_{n}\right) \in M$ iff $\Phi\left(t_{2}, t_{3}, \ldots, t_{n}\right) \in M$. As the equations of $M$ do not depend on $\left(w_{1}, \bar{w}_{1}\right)$, it follows that $\underline{\Phi}\left(0, t_{3}, \ldots, t_{n}\right) \in \underline{M}$ iff $\underline{\Phi}\left(t_{2}, t_{3}, \ldots, t_{n}\right) \in \underline{M}$. Consequently the vector field $\underline{L}_{2}:=\Phi_{*}\left(\partial / \partial t_{2}\right)$ is tangent to $\underline{M}$. Further, the foliation $\mathcal{F}_{L_{2}}$ is algebraic, since $\Phi$ is. In the coordinates over $\mathbb{C}^{n-1}$ defined by $\underline{\Phi}$, we have $\underline{L}_{2}=\partial / \partial t_{2}$. As above, we deduce that the equations of $\underline{M}$ are independent of $\left(w_{2}, \bar{w}_{2}\right)$, whence $M=\Delta^{2} \times \underline{M}$, with $\underline{M} \subset \mathbb{C}^{n-2}$ being CR-generic. If $\kappa \geq 3$, we proceed analogously, etc. up to $L_{\kappa}$, which completes the proofs of Lemma 9.11 and of Theorem 9.10.

9.13. Holomorphic degeneracy. - Let $\Sigma$ be an irreducible real algebraic subset of $\mathbb{C}^{n}$. Let $p \in \Sigma_{\mathrm{CR}}$. Let $\kappa_{\Sigma, p}^{\text {hol }}$ denote the maximal number of $(1,0)$ vector fields $L_{1}, \ldots, L_{j}$ with holomorphic coefficients which are tangent to $\Sigma_{\mathrm{CR}} \cap \mathcal{V}_{\mathbb{C}^{n}}(p)$ and linearly independent over $\mathcal{H}\left(\mathcal{V}_{\mathbb{C}^{n}}(p)\right)$ (cf. [2], [14]). Clearly, we have $\kappa_{\Sigma, p}^{\text {hol }} \geq \kappa_{\Sigma}$, since we do not require the induced foliations to be algebraic. Nevertheless, we can deduce from Theorem 2.5 that these two integers coincide.

Corollary 9.14. - For all $p \in \Sigma_{\mathrm{CR}}$, we have $\kappa_{\Sigma, p}^{\mathrm{hol}}=\kappa_{\Sigma, p}=\kappa_{\Sigma}$.

Proof. - Set $\chi:=\kappa_{\Sigma, p}^{\text {hol }}$. Let us choose $q \in \mathcal{V}_{\mathbb{C}^{n}}(p)$ with $L_{1}(q), \ldots, L_{\chi}(q)$ being linearly independent, in a neighborhood of which $\Sigma=\Delta^{\kappa_{\Sigma}} \times \underline{\Sigma}_{q}$. As $\chi>\kappa_{\Sigma}$, a linear combination of the $L_{j}$ 's is tangent to $0 \times \underline{\Sigma}_{q}$. We are thus reduced to prove the corollary in the case where $\kappa_{\Sigma}=0$ and $\chi \geq 1$. By contradiction, assume that $\kappa_{\Sigma}=0$ but there exists a holomorphic vector field $L$ with $L(p) \neq 0$ tangent to $\Sigma \cap \mathcal{V}_{\mathbb{C}^{n}}(p)$. Let

$$
\varphi(u, t):=\exp (u L)(t)
$$

be the flow of $L$, written in coordinates $t$ vanishing at $p$. Set

$$
u_{a, b}(t):=b+\sum_{k=1}^{n} a_{k} t_{k},
$$

with small $a \in \mathbb{C}^{n}$ and $b \in \mathbb{C}$. According to the separate algebraicity principle, $\varphi(u, t)$ is algebraic if and only if $t \mapsto \varphi\left(u_{a, b}(t), t\right)$ is algebraic for all $(a, b)$. If $\varphi(u, t)$ was algebraic, there would exist an algebraic biholomorphism straightening $L$ in $\partial / \partial t_{1}$ (see the proof of Lemma 9.12), whence $\kappa_{\Sigma} \geq 1$, which is untrue. Thus there exist $a$ and $b$ such that $t \mapsto \varphi\left(u_{a, b}(t), t\right)$ is a nonalgebraic biholomorphic self-map of $(\Sigma, p)$. Then Corollary 3.3 implies $\kappa_{\Sigma} \geq 1$, contradiction.

9.15. Link with the defining equations. - Finally, we describe a useful mean of calculating $\kappa_{\Sigma}(c f .[2],[14])$. Without loss of generality, we can pick a small connected piece $M$ of $\Sigma_{\mathrm{CR}}$ centered at one of its points $p$ at which 
$M$ is CR-generic and given by holomorphic algebraic equations $\bar{z}_{\ell}=Q_{\ell}(\bar{w}, t)$, $\ell=1, \ldots, d$. Let us write

$$
Q_{\ell}(\bar{w}, t)=\sum_{\beta \in \mathbb{N} m} \bar{w}^{\beta} Q_{\ell, \beta}(t) .
$$

Let $\mathcal{Q}(t)$ denote the $d \times \infty$ matrix of holomorphic algebraic functions $\left(Q_{\ell, \beta}(t)\right)_{1 \leq \ell \leq d, \beta \in \mathbb{N}^{m}}$. After a dilatation of coordinates, we can assume that all the $Q_{\ell, \beta}$ are holomorphic in $\Delta^{n}$. Let us study abstractly the situation where we are given such a denumerable collection

$$
\Phi(t):=\left(\varphi_{k}(t)\right)_{k \in \mathbb{N}} \in \mathbb{C}^{\infty}
$$

of holomorphic functions over $\Delta^{n}$. By the Jacobian Jac $\Phi(t)$ of $\Phi(t)$, we understand the $n \times \infty$ matrix

$$
\left(\frac{\partial \varphi_{k}}{\partial t_{\ell}}(t)\right)_{1 \leq \ell \leq n, k \in \mathbb{N}}
$$

Let $i$ be an integer with $1 \leq i \leq n$. We consider the denumerable collection of all $i \times i$ minors of Jac $\Phi(t)$. Let $\chi$ denote the generic rank of the map $\Phi(t)$. In other words, all the $(\chi+1) \times(\chi+1)$ minors of Jac $\Phi(t)$ vanish identically over $\Delta^{n}$ and there exists a $\chi \times \chi$ minor of Jac $\Phi(t)$ that does not vanish identically. We shall denote by gen-rk $\mathbb{C}_{\mathbb{C}} \Phi=\chi$ the generic rank of $\Phi$.

The set $E \subset \Delta^{n}$ of points where all the $\chi \times \chi$ minors vanish is then a proper real algebraic subset of $\Delta^{n}$. According to the constant rank theorem (also valuable for a denumerable collection of holomorphic functions), for each point $p \in \Delta^{n} \backslash E$, there exists a neighborhood $V$ of $p$ contained in $\Delta^{n} \backslash E$ such that for each $q \in V$, the set

$$
\mathcal{F}_{q}:=\left\{t \in V: \varphi_{k}(t)=\varphi_{k}(q), \forall k \in \mathbb{N}\right\}
$$

is a complex algebraic variety and the union of the $\mathcal{F}_{q}$ equips $V$ with a holomorphic algebraic foliation of dimension $n-\chi$. Applying this to $\mathcal{Q}(t)$, we deduce the following fundamental result.

THEOREM 9.16. - Let $M$ be a connected local piece of a real algebraic $C R$ generic submanifold of $\mathbb{C}^{n}$ of $C R$ dimension $m$ and of codimension $d$ passing through the origin which is given in coordinates $t=(w, z) \in \mathbb{C}^{m} \times \mathbb{C}^{d}$ by the $d$ scalar equations

$$
\bar{z}_{\ell}=Q_{\ell}(\bar{w}, t)=\sum_{\beta \in \mathbb{N} m} \bar{w}^{\beta} Q_{\ell, \beta}(t), \quad \ell=1, \ldots, d,
$$

let $\mathcal{Q}(t)$ denote the $d \times \infty$ matrix of holomorphic algebraic functions $\left(Q_{\ell, \beta}(t)\right)_{1 \leq \ell \leq d, \beta \in \mathbb{N}^{m}}$. Let $\chi_{M}$ denote the generic rank of $\mathcal{Q}(t)$. Then the degree of algebraic degeneracy $\kappa_{M}$ of $M$ defined in $\S 9.7$ above has the following properties:

1) the relations $\kappa_{M}+\chi_{M}=n$ and $\kappa_{M} \leq m$ hold;

TOME $129-2001-\mathrm{N}^{\mathrm{O}} 4$ 
2) the set of points $t \in M$ where the rank of $\mathcal{Q}(t)$ is $<\chi_{M}$ is a proper real algebraic subvariety of $M$.

Proof. - It remains only to check that $\kappa_{M}=n-\chi_{M}$. At first, applying Theorem 9.10, we see immediately that $\chi_{M} \leq n-\kappa_{M}$. Conversely, at a point $p$ close to the origin at which the matrix $\mathcal{Q}(t)$ is of constant rank, a neighborhood $V$ of $p$ algebraically foliates by $\left(n-\chi_{M}\right)$-dimensional leaves defined by

$$
\mathcal{F}_{q}=\left\{t \in V: Q_{\ell, \beta}(t)=Q_{\ell, \beta}(q), \forall \ell, \beta\right\} .
$$

Of course, such a foliation is algebraically biholomorphic to a product by a polydisc $\Delta^{n-\chi_{M}}$. It remains only to show that the foliation is tangent to $M$, namely that one of its leaf is entirely contained in $M$ if and only if it intersects $M$. But suppose $q \in M$, i.e. $z_{q}=\bar{Q}\left(w_{q}, \bar{t}_{q}\right)$, and let $t \in \mathcal{F}_{q}$ which by definition satisfies $\bar{Q}_{\ell, \beta}(\bar{t})=\bar{Q}_{\ell, \beta}(\bar{q})$ for all $\ell, \beta$. We deduce $z_{q}=\bar{Q}\left(w_{q}, \bar{t}\right)$. Remember that there exists an invertible $d \times d$ matrix $a(t, \tau)$ satisfying

$$
z-\bar{Q}(w, \tau) \equiv a(t, \tau)(\xi-Q(\zeta, t)) .
$$

We deduce $\bar{z}=Q\left(\bar{w}, t_{q}\right)$. Finally, $\bar{z}=Q(\bar{w}, t)$, again since $Q_{\ell, \beta}(t)=Q_{\ell, \beta}(q)$ for all $\ell, \beta$. Hence $t \in M$. This shows that $\kappa_{M} \geq n-\chi_{M}$, as desired.

\section{Segre-transversality}

10.1. Definitions. - Our goal in this paragraph is to establish that up to and including codimension two, Segre-transversality is equivalent to minimality, at Zariski-generic points. At first, we need preliminary definition. As above, let $M$ be a CR-generic submanifold of $\mathbb{C}^{n}$ with $m:=\operatorname{dim}_{\mathrm{CR}} M$ and $d=\operatorname{codim}_{\mathbb{R}} M$. We assume that $M$ is real analytic or real algebraic. Let $p \in M$. Let $S_{\bar{q}}$ denote the Segre varieties associated with $q \in \mathcal{V}_{\mathbb{C}^{n}}(p), c f$. (5.3). Remember that $q \in S_{\bar{p}}$ iff $p \in S_{\bar{q}}$. Following [5] and slightly refining the notions, we shall say that $M$ is called Segre-transversal at $p$ if for each neighborhood $V=\mathcal{V}_{\mathbb{C}^{n}}(p)$ of $p$, there exists $k \in \mathbb{N}_{*}$ and $p_{1}, \ldots, p_{k} \in S_{\bar{p}} \cap V$ such that

$$
T_{p} S_{\bar{p}_{1}}+\cdots+T_{p} S_{\bar{p}_{k}}=T_{p} \mathbb{C}^{n} .
$$

Also, $M$ is called Segre-transversal in $p$ if for each neighborhood $V=\mathcal{V}_{\mathbb{C}^{n}}(p)$ of $p$, there exist $k \in \mathbb{N}_{*}, q \in V, p_{1}, \ldots, p_{k} \in S_{\bar{q}} \cap V$ such that

$$
T_{q} S_{\bar{p}_{1}}+\cdots+T_{q} S_{\bar{p}_{k}}=T_{q} \mathbb{C}^{n} .
$$

Remarks. - 1) Obviously, Segre-transversality at $p$ implies Segre-transversality in $p$.

2) We observe that Segre-transversality at $p$ does not entail that the second Segre set ( cf. [2]) $S_{\bar{p}}^{2}:=\bigcup_{q \in S_{\bar{p} \cap V}} S_{\bar{q}} \cap V$ contains an open set in $\mathbb{C}^{n}$, as shows the following example in $\mathbb{C}^{3}, M: z_{1}=\bar{z}_{1}+i w \bar{w}, z_{2}=\bar{z}_{2}+i w^{2} \bar{w}^{2}$, or, more generally, as shows any $M$ of codimension $d>2 m$.

BULLETIN DE LA SOCIÉtÉ MATHÉMATIQUE DE FRANCE 
3) Nevertheless, it is known and clear that Segre-transversality at $p$ entails minimality at $p$, because it is easy to check in coordinates that all the Segre varieties $S_{\overline{p_{1}}}, \ldots, S_{\overline{p_{k}}}$ must be contained in the intrinsic complexification $\mathcal{O}_{p}^{i_{c}}$ of the CR orbit of $p$ (see also [2], [9], [13]), whence condition (10.2) forces $\mathcal{O}_{p}^{i_{c}}$ to be of dimension $n$.

Proposition 10.4. - The following properties hold:

1) A connected $C R$-generic manifold $M \subset \mathbb{C}^{n}$ of codimension 1 or 2 is minimal at a Zariski-generic point if and only if it is Segre-transversal in at least one point $p \in M$.

2) The $C R$-generic manifold $M \subset \mathbb{C}^{4}$ of codimension 3 given by

$$
z_{1}=\overline{z_{1}}+i w \bar{w}, \quad z_{2}=\bar{z}_{2}+i w \bar{w}\left(w^{2}+\bar{w}^{2}\right), \quad z_{3}=\bar{z}_{3}+i w^{3} \bar{w}^{3}
$$

is minimal at 0 but not Segre-transversal in any point.

Proof. - The example in part 2) is borrowed from [5]. Part 1) is new for the codimension $d=2$. In order to prove this proposition, we plan now to give characterizations of Segre-transversalities. In fact, a characterization of Segretransversality at a point is given in [5], but for $M$ rigid algebraic only. We seek a general formulation. Thus, we assume that $p=0, V=\Delta^{n}$ and that $M$ is given by (6.3). Let $q \in \Delta^{n}$, let $k \in \mathbb{N}^{*}$, let $p_{1}, \ldots, p_{k} \in S_{\bar{q}} \cap \Delta^{n}$, i.e.

$$
z_{p_{j}}=\bar{z}_{q}+i \bar{\Theta}\left(w_{p_{j}}, \bar{w}_{q}, \bar{z}_{q}\right), \quad j=1, \ldots, k .
$$

To compute $T_{q} S_{\bar{p}_{j}}$ is easy: $T_{q} S_{\bar{p}_{j}}$ is in fact generated by the $m$-vector field (cf. $(6.2))$

$$
\mathcal{L}=\frac{\partial}{\partial w}+i \bar{\Theta}_{w}\left(w_{q}, \bar{w}_{p_{j}}, z_{q}-i \Theta\left(\bar{w}_{p_{j}}, w_{q}, z_{q}\right)\right) \frac{\partial}{\partial z}
$$

i.e. by a collection $v^{\ell}=v^{\ell}\left(w_{q}, z_{q}, \bar{w}_{p_{j}}\right)$ of vectors $\ell=1, \ldots, m$ of the form

$$
\left(0, \ldots, 1, \ldots, 0, v_{1}^{\ell}\left(w_{q}, z_{q}, \bar{w}_{p_{j}}\right), \ldots, v_{d}^{\ell}\left(w_{q}, z_{q}, \bar{w}_{p_{j}}\right)\right) \in \mathbb{C}^{m} \times \mathbb{C}^{d},
$$

with 1 at the $m$-th place, $j=1, \ldots, k$. Let us denote by $\Lambda\left(w_{q}, z_{q}, \bar{w}_{p_{j}}\right)$ the linear space generated by the $v^{\ell}$, for $\ell=1, \ldots, m$. By definition, $M$ is Segre-transversal in $p$ if and only if there exists $q$ arbitrarily close to $p$ and $p_{1}, \ldots, p_{k} \in S_{\bar{q}}$ such that

$$
\operatorname{Span}\left(\Lambda\left(w_{q}, z_{q}, \bar{w}_{p_{1}}\right), \ldots, \Lambda\left(w_{q}, z_{q}, \bar{w}_{p_{k}}\right)\right)=\mathbb{C}^{n} .
$$

Of course it suffices to choose $k=d+1$ to test (10.8). We can also complexify (10.8) and get

$$
\operatorname{Span}\left(\Lambda\left(w, z, \zeta_{1}\right), \ldots, \Lambda\left(w, z, \zeta_{d+1}\right)\right)=\mathbb{C}^{n} .
$$

Introducing all $n \times n$ minors of the system of $m(d+1)$ vectors $v^{\ell}\left(w, z, \zeta_{j}\right)$, $\ell=1, \ldots, m, j=1, \ldots, d+1$, denoting by $N$ the number of such minors, we 
may build a holomorphic map

$$
\mathbb{X}: \mathcal{M}_{\natural}^{d+1} \longrightarrow \mathbb{C}^{N}
$$

whose components are these minors and which satisfies $\mathbb{X}(t, 0, \ldots, t, 0) \equiv 0$. We may equip with a projection $\pi_{\natural}: \mathcal{M}_{\natural}^{d+1} \rightarrow \mathbb{C}_{t}^{n}$ the set

$$
\mathcal{M}_{\natural}^{d+1}=\left\{\left(t_{1}, \tau_{1}, \ldots, t_{d+1}, \tau_{d+1}\right) \in \mathcal{M}^{d+1}: t_{i}=t_{j}, 1 \leq i, j \leq d+1\right\}
$$

such that $(10.9)$ holds at $(w, z)$ if and only if there exists $p_{\natural} \in \pi_{\natural}^{-1}(w, z)$ with $\mathbb{X}\left(p_{\natural}\right) \neq 0$. Consequently $(10.9)$ holds at $t=(w, z)$ if and only if

$$
\mathbb{X}\left(t, \zeta_{1}, \ldots, t, \zeta_{d+1}\right) \not \equiv_{\zeta_{1}, \ldots, \zeta_{d+1}} 0 \in \mathbb{C}^{N} .
$$

Now writing

$$
\mathbb{X}\left(t, \zeta_{1}, \ldots, t, \zeta_{d+1}\right)=\sum_{\gamma_{\natural} \in \mathbb{N}_{*}^{m}(d+1)} \zeta_{\natural}^{\gamma_{\natural}} \mathbb{X}_{\gamma_{\natural}}(w, z), \quad \mathbb{X}_{\gamma_{\natural}}(w, z) \in \mathbb{C}^{N},
$$

we have got that there exist $\gamma_{\natural} \in \mathbb{N}_{*}^{m(d+1)}$ and $(w, z) \in \Delta^{n}$ such that $\mathbb{X}_{\gamma_{\sharp}}(w, z) \neq 0$. As a usual consequence of analyticity (here of $\mathbb{X}$ ) we see that (10.9) holds at $\left(w_{q}, z_{q}\right)$ if and only if (10.9) holds for every $(w, z) \in \Delta^{n}$ minus a proper complex analytic subset. We thus have proved:

Proposition 10.13. - A connected real analytic or real algebraic CR-generic manifold $M$ is Segre-transversal in a point if and only if it is Segre-transversal in every point. In that case $M$ is Segre-transversal at every point outside a proper real analytic or real algebraic subvariety. In this case, $M$ is be called Segre-transversal.

Proof of Proposition 10.4 1). - If $d=1$, it is very easy to check that $M$ is Segre-transversal if and only if $M$ is not Levi-flat if and only if it is minimal at a Zariski-generic point, $c f$. [5]. Thus, let $d=2$ and assume that $M$ is minimal at a Zariski-generic point. To simplify the notation, let us assume that $m=1$. Then at a Zariski-generic point $p \in M$, the vector-valued Levi-form of $M$ has rank 1. Further, the second order Lie brackets of $T^{c} M$ must complete the tangent space to $M$ at a Zariski-generic point (otherwise, the distribution spanned by $T^{c} M$ and its first order Lie brackets is involutive and then $M$ locally foliates in three-dimensional CR orbits, contradiction). Thus, there are holomorphic coordinates $\left(w, z_{1}, z_{2}\right)$ vanishing at such a Zariski-generic point $p$ in which the equations of $M$ are

$$
\begin{aligned}
& z_{2}=\bar{z}_{2}+i w \bar{w}\left[1+O(|w|)+O\left(\bar{z}_{2}, \bar{z}_{3}\right)\right], \\
& z_{3}=\bar{z}_{3}+i w \bar{w}\left[w+\bar{w}+O\left(|w|^{2}\right)+O\left(\bar{z}_{2}, \bar{z}_{3}\right)\right] .
\end{aligned}
$$

Choosing then a point $q=\left(w_{q}, 0,0\right)$ with $w_{q} \neq 0$ small, it is easy to check that condition (10.8) is satisfied for suitable points $p_{1}, p_{2}, p_{3} \in S_{\bar{q}}$ with $w_{p_{1}}=0$, 
and $w_{p_{2}}, w_{p_{3}}$ small. The general case $m \geq 2$ is similar or can be reduced to the case $m=1$ by slicing. This completes the proof of Proposition 10.4.

\section{Algebraicity of the reflection mapping and transcendence degree of the mapping}

11.1. Introduction. - Now, to end-up this article, we come to the description of an equivalent formulation of our main Theorem 2.5. An interesting invariant of which to show analyticity (in case $f$ is in $\mathcal{C}_{\mathrm{CR}}^{\infty}$ and $M, M^{\prime}$ are real analytic) or algebraicity (in case $f$ is already holomorphic, and $M, M^{\prime}$ are real algebraic), is the so-called 'reflection mapping' (see [11], where the interest of its study with no nondegeneracy condition on $M^{\prime}$ was pointed out for the first time). The reflection mapping is the holomorphic map

$$
\mathbb{C}^{n} \times \mathbb{C}^{n^{\prime}} \ni\left(t, \tau^{\prime}\right) \longmapsto \xi^{\prime}-Q^{\prime}\left(\zeta^{\prime}, f(t)\right) \in \mathbb{C}^{d^{\prime}},
$$

where $\bar{z}^{\prime}=Q^{\prime}\left(\bar{w}^{\prime}, t^{\prime}\right)$ is a local system of $d^{\prime}$ defining equations for $M^{\prime}$ as in $\S 6.1$ above. Let us denote it by $\mathcal{R}_{f}^{\prime}\left(t, \tau^{\prime}\right)$, where $\tau^{\prime}=\left(\zeta^{\prime}, \xi^{\prime}\right)$. Clearly, $\mathcal{R}_{f}^{\prime}$ is relatively algebraic with respect to $\tau^{\prime}$ and a priori only holomorphic with respect to $t$.

TheOREM 11.2 (case $d=1$ : see [12]). - Let $h: M \rightarrow M^{\prime}$ be a holomorphic map of generic rank $n$ between two small pieces of connected somewhere minimal CR-generic real algebraic submanifolds of $\mathbb{C}^{n}$ of the same $C R$ dimension $m \geq 1$ and of codimension $d \geq 1$. Then the reflection mapping $\mathcal{R}_{h}^{\prime}$ associated with $h$ and an arbitrary local system of coordinates for $M^{\prime}$ as above is complex algebraic with respect to both variables $\left(t, \tau^{\prime}\right)$.

Proof. - Using the biholomorphic invariance of Segre varieties, one can check that the algebraicity of $\mathcal{R}_{h}^{\prime}$ is preserved under changes of algebraic coordinates fixing a point and under small shifts of a center point $p^{\prime} \in M^{\prime}$ (exercise). Thus, after a slight delocalization, namely after an arbitrarily small shift of the center point, and after a change of algebraic coordinates as in Theorem 9.10, we can assume that $f:(M, p) \rightarrow\left(M^{\prime}, p^{\prime}\right)$ is a local biholomorphic mapping with $M^{\prime}=\Delta^{\kappa^{\prime}} \times \underline{M}^{\prime}, \kappa^{\prime}:=\kappa_{M^{\prime}}$. Composing $f$ with a projection, we get a submersive local holomorphic map $\underline{f}: M \rightarrow \underline{M}^{\prime}$, with $\underline{M}^{\prime}$ minimal for inclusion. Since $\kappa_{\underline{M}^{\prime}}=0$, Theorem 3.1 yields that $f$ is algebraic. Notice that there exist coordinates

$$
t^{\prime}=\left(w^{\prime}, z^{\prime}\right)=\left(u^{\prime}, v^{\prime}, z^{\prime}\right) \in \mathbb{C}^{m^{\prime}-\kappa^{\prime}} \times \mathbb{C}^{\kappa^{\prime}} \times \mathbb{C}^{d^{\prime}}
$$

as in $\S 6.1$ such that the equations of $\left(M^{\prime}, p^{\prime}\right)$ are independent of $\left(v^{\prime}, \bar{v}^{\prime}\right)$, namely $\bar{z}^{\prime}=Q^{\prime}\left(\bar{u}^{\prime}, u^{\prime}, z^{\prime}\right)$. Let $\left(u^{\prime}, v^{\prime}, z^{\prime}\right)^{c}=:\left(\mu^{\prime}, \nu^{\prime}, \xi^{\prime}\right)$. Finally, the reflection mapping $\mathcal{R}_{f}^{\prime}\left(t, \tau^{\prime}\right)=\xi^{\prime}-Q^{\prime}\left(\mu^{\prime}, \underline{f}(t)\right) \in \mathbb{C}^{d^{\prime}}$ is then obviously algebraic, because $\underline{f}$ is. The proof of Theorem $\overline{11} .2$ is complete.

TOME $129-2001-\mathrm{N}^{\mathrm{O}} 4$ 
11.3. Algebraicity of the reflection mapping with no rank assumption. - More generally, by similar arguments, we shall now establish that the algebraicity of the reflection mapping associated with $f: M \rightarrow M^{\prime}$ is in fact equivalent to Theorem 2.5. An important assumption will be that $M^{\prime}$ is here minimal for inclusion containing $f(M)$.

TheOREm 11.4. - Let $f$ be a local holomorphic map $(M, p) \rightarrow\left(M^{\prime}, p^{\prime}\right)$ between two real algebraic CR-generic manifolds with $(M, p)$ minimal at a Zariskigeneric point, with $\left(M^{\prime}, p^{\prime}\right)$ minimal for inclusion containing $f(M, p)$ and given by $d^{\prime}$ equations of the form $\bar{z}_{\ell}^{\prime}=Q_{\ell}^{\prime}\left(\bar{w}^{\prime}, t^{\prime}\right), \ell=1, \ldots, d^{\prime}$. Then the reflection mapping:

$$
\left(\mathbb{C}^{n}, p\right) \times\left(\mathbb{C}^{n^{\prime}}, \bar{p}^{\prime}\right) \ni\left(t, \bar{\nu}^{\prime}\right) \mapsto\left(\xi_{\ell}^{\prime}-Q_{\ell}^{\prime}\left(\zeta^{\prime}, h(t)\right)\right)_{1 \leq \ell \leq d^{\prime}} \in \mathbb{C}^{d^{\prime}}
$$

extends as an algebraic map of $\left(t, \tau^{\prime}\right) \in \mathbb{C}^{n} \times \mathbb{C}^{n^{\prime}}$. Conversely, if the abovedefined reflection mapping is algebraic, then $\nabla^{\operatorname{tr}}(f) \leq \kappa_{M^{\prime}}$.

Proof. - Since $M^{\prime}$ is minimal for inclusion and since the exceptional set of points $q^{\prime}$ in a neighborhood of which $M^{\prime} \cap \mathcal{V}_{\mathbb{C}^{n^{\prime}}}\left(q^{\prime}\right)$ is not equivalent to a product $\Delta^{\kappa_{M^{\prime}}} \times \underline{M}^{\prime}$ is a proper real algebraic subvariety of $M^{\prime}$ (cf. Theorems 9.10 and 9.16), then $f(M)$ encounters in fact the set of points $q^{\prime}$ for which $M^{\prime} \cap \mathcal{V}_{\mathbb{C}^{n^{\prime}}}\left(q^{\prime}\right) \cong_{\mathcal{A}} \Delta^{\kappa_{M^{\prime}}} \times \underline{M}^{\prime}$. As in the proof of Theorem 11.2, we then deduce the algebraicity of $\mathcal{R}_{f}^{\prime}$.

Conversely, let $M^{\prime}$ be minimal for inclusion, let $M^{\prime}=\Delta^{\kappa^{\prime}} \times \underline{M}^{\prime}$ in a neighborhood of $q^{\prime}$ close to $p^{\prime}$, with $\kappa^{\prime}:=\kappa_{M^{\prime}}$, choose coordinates $\left(u^{\prime}, v^{\prime}, z^{\prime}\right)$ vanishing at $q^{\prime}$ as above and assume that $\xi^{\prime}-Q^{\prime}\left(\mu^{\prime}, \underline{f}(t)\right)$ is algebraic. Differentiating with respect to $\mu^{\prime}$, we deduce that the functions

$$
\frac{1}{\beta !}\left[\partial_{\mu^{\prime}}^{\beta} Q^{\prime}\left(\mu^{\prime}, \underline{f}(t)\right)\right]_{\mu^{\prime}=0}=: Q_{\beta}^{\prime}(\underline{f}(t)), \quad \beta \in \mathbb{N}^{m^{\prime}-\kappa^{\prime}},
$$

are algebraic. By Theorem 9.16, 2), it follows from the assumption $\kappa_{\underline{M}^{\prime}}=0$ that the set $E^{\prime}$ of points $\left(u^{\prime}, z^{\prime}\right) \in \underline{M}^{\prime}$ at which all $d^{\prime} \times d^{\prime}$ minors of the Jacobian matrix of

$$
\mathcal{Q}^{\prime}\left(t^{\prime}\right)=\left(Q_{\ell, \beta}^{\prime}\left(u^{\prime}, z^{\prime}\right)\right)_{1 \leq \ell \leq d^{\prime}, \beta \in \mathbb{N}^{m^{\prime}-\kappa^{\prime}}}
$$

vanish is a proper real algebraic subvariety of $M^{\prime}$. By minimality for inclusion of $M^{\prime}$, it follows that $f(M, p)$ is not contained in $E^{\prime}$. Using then the algebraic implicit function theorem we deduce that $\underline{f}$ is algebraic. This implies that $\nabla^{\operatorname{tr}}(f) \leq \kappa^{\prime}$, as desired.

11.6. Algebraic approximation. - Let us conclude this paper with a nice application of Theorem 11.4. We need some preliminary. Under the assumptions of Theorem 11.4, let $t \in \mathbb{C}^{n}, t^{\prime} \in \mathbb{C}^{n^{\prime}}$ be coordinates vanishing at $p, p^{\prime}$. Let us denote

$$
r(t, \tau):=z-\bar{Q}(w, \tau) \quad \text { and } \quad r^{\prime}\left(t^{\prime}, \tau^{\prime}\right):=z^{\prime}-\bar{Q}^{\prime}\left(w^{\prime}, \tau^{\prime}\right),
$$

BULLETIN DE LA SOCIÉtÉ MATHÉMATIQUE DE FRANCE 
whence $\bar{r}(\tau, t)=\xi-Q(\zeta, t)$ and $\bar{r}^{\prime}\left(\tau^{\prime}, t^{\prime}\right)=\xi^{\prime}-Q^{\prime}\left(\zeta^{\prime}, t^{\prime}\right)$. Because $M$ and $M^{\prime}$ are real, there exist a $d \times d$ matrix $a(t, \tau)$ of holomorphic algebraic functions with $a(0)=-I_{d \times d}$ and $r(t, \tau) \equiv a(t, \tau) \bar{r}(\tau, t)$, and similarly $a^{\prime}\left(t^{\prime}, \tau^{\prime}\right)$ with $a^{\prime}(0)=-I_{d^{\prime} \times d^{\prime}}$ and $r^{\prime}\left(t^{\prime}, \tau^{\prime}\right) \equiv a^{\prime}\left(t^{\prime}, \tau^{\prime}\right) \bar{r}^{\prime}\left(\tau^{\prime}, t^{\prime}\right)$. Since $(M, p)$ is mapped in $\left(M^{\prime}, p^{\prime}\right)$, there exist a $d^{\prime} \times d$ matrix $b(t, \tau)$ of holomorphic functions such that $r^{\prime}(f(t), \bar{f}(\tau)) \equiv b(t, \tau) r(t, \tau)$. Here, $f(t) \in \mathbb{C}\{t\}^{n^{\prime}}$ is a power series vanishing at 0 . Finally, we set

$$
Q^{\prime}\left(\zeta^{\prime}, t^{\prime}\right):=\sum_{\beta \in \mathbb{N}^{\prime}} \zeta^{\prime \beta} Q_{\beta}^{\prime}\left(t^{\prime}\right) .
$$

Then it follows from Theorem 11.4 that for each $\beta \in \mathbb{N}^{m^{\prime}}$, the function

$$
\varphi_{\beta}^{\prime}(t):=Q_{\beta}^{\prime}(f(t))=\frac{1}{\beta !}\left[\partial_{\zeta^{\prime}}^{\beta} Q^{\prime}\left(\zeta^{\prime}, f(t)\right)\right]_{\zeta^{\prime}=0}
$$

is algebraic. By applying the algebraic approximation theorem of Artin [1] to the collection of holomorphic algebraic equations $Q_{\beta}^{\prime}\left(t^{\prime}\right)-\varphi_{\beta}^{\prime}(t)=0$, we deduce that for each integer $N \in \mathbb{N}_{*}$, there exists a holomorphic algebraic mapping $F:\left(\mathbb{C}^{n}, p\right) \rightarrow\left(\mathbb{C}^{n^{\prime}}, p^{\prime}\right)$, namely $F(t) \in \mathbb{C}\{t\}^{n^{\prime}}$, which is algebraic, such that $Q_{\beta}^{\prime}(F(t))=\varphi_{\beta}^{\prime}(t)$ for all $\beta \in \mathbb{N}^{m^{\prime}}$ and $F(t) \equiv f(t)\left(\bmod |t|^{N}\right)$. We observe that it follows that $F$ maps $(M, p)$ in $\left(M^{\prime}, p^{\prime}\right)$.

LEMma 11.7. - Suppose that $F(t) \in \mathbb{C}\{t\}^{n^{\prime}}$ satisfies $Q_{\beta}^{\prime}(F(t))=Q_{\beta}^{\prime}(f(t))$. Then $r^{\prime}(F(t), \bar{F}(\zeta, Q(\zeta, t))) \equiv 0$ in $\mathbb{C}\{\zeta, t\}^{n^{\prime}}$.

Proof. - Indeed, if we denote $f=(g, h) \in \mathbb{C}^{m^{\prime}} \times \mathbb{C}^{d^{\prime}}$, we have first

$$
\bar{h}(\zeta, Q(\zeta, t)) \equiv \sum_{\beta \in \mathbb{N}^{m^{\prime}}} \bar{g}(\zeta, Q(\zeta, t))^{\beta} Q_{\beta}^{\prime}(F(t)) .
$$

Let us write $F=(G, H) \in \mathbb{C}^{m^{\prime}} \times \mathbb{C}^{d^{\prime}}$. First, from the relation

$$
r^{\prime}(F(t), \bar{f}(\tau)) \equiv a^{\prime}(F(t), \bar{f}(\tau)) \bar{r}^{\prime}(\bar{f}(\tau), F(t)),
$$

we deduce $H(t) \equiv \sum_{\beta \in \mathbb{N}^{\prime}} G(t)^{\beta} \bar{Q}_{\beta}^{\prime}(\bar{f}(\tau))$. As the coordinates $(\zeta, t)$ and $(w, \tau)$ are equivalent on $\mathcal{M}$, we deduce

$$
H(w, \bar{Q}(w, \tau)) \equiv \sum_{\beta \in \mathbb{N}^{m^{\prime}}} G(w, \bar{Q}(w, \tau))^{\beta} \bar{Q}_{\beta}^{\prime}(\bar{f}(\tau)) .
$$

Finally, we get $H(w, \bar{Q}(w, \tau)) \equiv \sum_{\beta \in \mathbb{N}^{m^{\prime}}} G(w, \bar{Q}(w, \tau))^{\beta} \bar{Q}_{\beta}^{\prime}(\bar{F}(\tau))$, as desired.

In particular, we obtain the following interesting application. Let $f$ : $(M, p) \rightarrow\left(M^{\prime}, p^{\prime}\right)$ be a biholomorphic equivalence between two real algebraic CR-generic manifolds such that $(M, p)$ is minimal at a Zariski-generic point. Then there exists an algebraic holomorphic equivalence $F:(M, p) \rightarrow\left(M^{\prime}, p^{\prime}\right)$. Is such a property also true for nowhere minimal CR-generic manifolds?

TOME $129-2001-\mathrm{N}^{\mathrm{O}} 4$ 


\section{BIBLIOGRAPHY}

[1] ARTin (M.) - Algebraic approximation of structures over complete local rings, Inst. Hautes Études Sci. Publ. Math., t. 36 (1969), pp. 23-58.

[2] Baouendi (M. S.), Ebenfelt (P.) \& Rothschild (L. P.) - Algebraicity of holomorphic mappings between real algebraic sets in $\mathbb{C}^{n}$, Acta Math., t. 177 (1996), no. 2, pp. 225-273.

[3] Bochner (S.) \& Martin (W.) - Several complex variables, in Princeton Math. Ser., vol. 10, Princeton Univ. Press, Princeton, N.J., 1949.

[4] Chirka (E.) - An introduction to the geometry of CR manifolds, Russian Math. Surveys, t. 46 (1991), no. 1, pp. 95-197.

[5] Coupet (B.), Meylan (F.) \& Sukhov (A.) - Holomorphic maps of algebraic CR manifolds, Int. Math. Research Notices, t. 1 (1999), pp. 1-29.

[6] Coupet (B.), Pinchuk (S.) \& Sukhov (A.) - On the partial analyticity of CR mappings, Math. Z., t. 235 (2000), pp. 541-557.

[7] Damour (S.) - Sur l'algébricité des applications holomorphes, C. R. Acad. Sci. Paris Sér. I Math., t. 332 (2001), no. 6, pp. 491-496.

[8] HuAnG (X.) - On the mapping problem for algebraic real hypersurfaces in the complex spaces of different dimension, Ann. Inst. Fourier Grenoble, t. 44 (1994), pp. 433-463.

[9] Merker (J.) - Vector field construction of Segre sets, e-print : http://arxiv.org/abs/math.cv/9901010.

[10] Note on double reflection and algebraicity of holomorphic mappings, Ann. Fac. Sci. Toulouse Math. (6), t. 9 (2000), no. 4, pp. 689-721.

[11] Merker (J.) \& MeYlan (F.) - On the Schwarz symmetry principle in a model case, Proc. Amer. Math. Soc., t. 127 (1999), pp. 1197-1102.

[12] Mir (N.) - Germs of holomorphic mappings between real algebraic hypersurfaces, Ann. Inst. Fourier Grenoble, t. 48 (1998), pp. 1025-1043.

[13] Sharipov (R.) \& Sukhov (A.) - On CR mappings between algebraic Cauchy-Riemann manifolds and separate algebraicity for holomorphic functions, Trans. Amer. Math. Soc., t. 348 (1996), pp. 767-780.

[14] Stanton (N.) - Infinitesimal CR automorphisms of real hypersurfaces, Amer. J. Math., t. 118 (1996), pp. 209-233.

[15] SukHov (A.) - On the mapping problem for quadric Cauchy-Riemann manifolds, Indiana Univ. Math. J., t. 42 (1993), pp. 27-32.

[16] Sussmann (H. J.) - Orbits of families of vector fields and integrability of distributions, Trans. Amer. Math. Soc., t. 180 (1973), pp. 171-188.

[17] Webster (S. M.) - On the mapping problem for algebraic real hypersurfaces, Invent. Math., t. 43-1 (1977), pp. 53-68.

[18] ZAITSEV (D.) - Algebraicity of local holomorphisms between real algebraic submanifolds in complex spaces, Acta Math., t. 183 (1999), pp. 273-305. 